\title{
Role of Synaptic and Intrinsic Membrane Properties in Short-Term Receptive Field Dynamics in Cat Area 17
}

\author{
Lionel G. Nowak, Maria V. Sanchez-Vives, and David A. McCormick \\ Department of Neurobiology and the Kavli Institute for Neuroscience, Yale University School of Medicine, New Haven, Connecticut 06510
}

We examined the mechanisms through which the prolonged presentation of either a high-contrast stimulus or an artificial scotoma [equivalent to the stimulation of the receptive field (RF) surround] induces changes in the RF properties of neurons intracellularly recorded in cat primary visual cortex. Discharge and synaptic RFs were quantitatively characterized using bright and dark bars randomly flashed in various positions. Compared with the lack of stimulation ( $0 \%$ contrast for $15-30 \mathrm{~s})$, stimulation with high-contrast sine-wave gratings (15-30 s) was followed by a strong reduction in gain and a weak but significant reduction in width of spike discharge RFs. These reductions were accompanied by a membrane potential hyperpolarization, a decrease of synaptic RF width, and varying changes of synaptic RF gain. Passive hyperpolarization by DC injection also produced significant reduction in the width and gain of discharge RF. Mimicking, in single neurons, high-contrast stimulation with high-intensity current injection also induced a membrane potential hyperpolarization, whose amplitude was correlated with discharge RF gain and width changes. Recovery from adaptation to high-contrast stimulation during the period of gray screen or scotoma presentation was associated with an increase in gain and discharge RF size. Stimulation of the RF surround with an artificial scotoma did not have any additional aftereffects over those of adaptation to a gray screen, indicating that the contraction and expansion of RF gain and size are attributable to intrinsic and synaptic mechanisms underlying adaptation and de-adaptation to strong visual stimuli.

Key words: cortex; receptive field; vision; visual; dynamics; plasticity; gain control

\section{Introduction}

The receptive field (RF) is defined as the region of sensory space in which a stimulus can modify action potential firing rate (Hartline, 1938; Kuffler, 1953). Instead of being static, the size and gain of RFs are flexible, being influenced by both the spatial configuration of stimuli and the recent history of sensory stimulation. Recent studies in visual cortex showed that, in the spatial domain, the region over which neurons respond is larger for low-contrast, compared with high-contrast, stimuli (Sengpiel et al., 1997; Kapadia et al., 1999; Sceniak et al., 1999; Anderson et al., 2001; Cavanaugh et al., 2002).

In the temporal domain, stimulation with artificial scotomas, in which the surround of the RF, but not the RF itself, is stimulated for several seconds to several minutes, has been reported to have varying consequences: some studies reported a large expan-

Received Sept. 20, 2004; revised Dec. 22, 2004; accepted Dec. 26, 2004.

This research was supported by National Institutes of Health (D.A.M.), Centre National de la Recherche Scientifique (L.G.N.) and Human Frontier Science Program, and Ministerio de Ciencia y Tecnología (M.V.S.-V.). We thank Bilal Haider and Alvaro Duque for their help in the last experiments and Jean Bullier and Ralph Freeman for critical comments on a previous version of this manuscript.

Correspondence should be addressed to David A. McCormick, Department of Neurobiology and the Kavli Institute for Neuroscience, Yale University School of Medicine, 333 Cedar Street, New Haven, CT 06510. E-mail: david.mccormick@yale.edu.

L. G. Nowak's present address: Centre de Recherche Cerveau et Cognition, Centre National de la Recherche Scientifique-Université Paul Sabatier, Unité Mixte de Recherche 5549, 133 Route de Narbonne, 31062 Toulouse Cedex, France. E-mail: lionel.nowak@cerco.ups-tlse.fr.

M. V. Sanchez-Vives's present address: Instituto de Neurociencias, Universidad Miguel Hernández-Consejo Superior de Investigaciones Científicas, Aptdo 18, 03550 San Juan de Alicante, Spain. E-mail: mavi.sanchez@umh.es. DOI:10.1523/JNEUROSCI.3897-04.2005

Copyright $\odot 2005$ Society for Neuroscience $\quad 0270-6474 / 05 / 251866-15 \$ 15.00 / 0$ sion of the RFs inside the scotoma, with this expansion being reversed with vigorous RF stimulation (Pettet and Gilbert, 1992; Das and Gilbert, 1995b); other studies reported a change in gain, but not size (DeAngelis et al., 1995), or no change at all in either gain or size (De Weerd et al., 1995).

Also in the temporal domain, changes in response gain are well known to occur with direct RF stimulation: during adaptation to a high-contrast stimulus, the response by a neuron progressively decreases, and this depression persists for seconds to tens of seconds after adaptation (Maffei et al., 1973; Albrecht et al., 1984). These effects are mediated in part by a hyperpolarization of the membrane potential (Carandini and Ferster, 1997; Sanchez-Vives et al., 2000a) and can be mimicked with intracellular current injection. This suggests that high-contrast adaptation and its aftereffects result, at least partially, from the activation of intrinsic $\mathrm{K}^{+}$currents (Sanchez-Vives et al., 2000b).

Visual cortical neurons receive visually evoked synaptic potentials from a region of visual space that is larger than the size of the "discharge" RF (Grinvald et al., 1994; Das and Gilbert, 1995a; Kitano et al., 1995; Toth et al., 1996; Bringuier et al., 1999). We hypothesized that, through an "iceberg effect," the hyperpolarization associated with high-contrast adaptation may result in a reduction in RF size and gain. Conversely, withdrawal of this hyperpolarization may result in apparent RF expansion. We tested this hypothesis on intracellularly recorded neurons in cat primary visual cortex by examining RF gain and size after adaptation to a gray screen (i.e., no patterned visual stimuli), an artificial scotoma, or a high-contrast visual stimulus. We demon- 
strate here that the gain, and to a lesser extent, the width of discharge RFs is larger after adaptation to an artificial scotoma or gray screen compared with after adaptation to a high-contrast visual stimulus. However, we found no significant difference between adaptation to a scotoma and adaptation to a gray screen. These differences in RF size and gain occurred in part through changes in the synaptic inputs to the recorded neurons and in part through adaptation-dependent changes in membrane potential.

\section{Materials and Methods}

Animal preparation. The protocols for animal preparation, electrophysiological recordings, and data acquisition have been detailed previously (Sanchez-Vives et al., 2000a). Anesthesia was induced in adult cats (2.5$3.5 \mathrm{~kg}$ ) with ketamine (12-15 mg/kg, i.m.) and xylazine ( $1 \mathrm{mg} / \mathrm{kg}$, i.m.). Atropine $(0.05 \mathrm{mg} / \mathrm{kg}$, s.c.) was given to reduce secretions. A forelimb vein was cannulated for intravenous perfusion, a tracheal tube was inserted for active ventilation, and wires were placed through the skin for electrocardiogram (ECG) recording. The cat was mounted in a stereotaxic frame and ventilated with a mixture of nitrous oxide and oxygen (2:1) with halothane (1.5\% during surgery). Two wires were inserted over the frontal cortex for epidural EEG recording. To minimize movements resulting from respiration and heart pulsation, a cisternal drainage and a bilateral pneumothorax were performed, and the animal was suspended by the rib cage to the stereotaxic frame. A craniotomy (3-4 mm wide) was made over the area centralis representation of area 17. After surgery, the animals were paralyzed with either Pavulon $(0.3 \mathrm{mg} / \mathrm{kg}$ for induction, followed by a continuous perfusion of $0.3 \mathrm{mg} \cdot \mathrm{kg}^{-1} \cdot \mathrm{h}^{-1}$ ) or Norcuron $(0.15 \mathrm{mg} / \mathrm{kg}$ for induction, followed by a continuous perfusion of $\left.0.1 \mathrm{mg} \cdot \mathrm{kg}^{-1} \cdot \mathrm{h}^{-1}\right)$. The nictitating membranes were retracted using ophthalmic phenylephrine, and the pupils were dilated and accommodation paralyzed with ophthalmic atropine. Area centralis and the optic discs were localized by back projection. The eyes were focused onto a computer monitor at $114 \mathrm{~cm}$ using corrective, gas-permeable contact lenses. No artificial pupils were used. During recording, anesthesia was maintained with $0.4-1 \%$ halothane in nitrous oxide/oxygen $(2: 1)$. The heart rate, expiratory $\mathrm{CO}_{2}$ concentration, rectal temperature, and blood $\mathrm{O}_{2}$ concentration were monitored throughout the experiment and maintained at $150-180$ beats per minute, $3-4 \%, 37-38^{\circ} \mathrm{C}$, and $>95 \%$ respectively. The state of the animal was monitored regularly through the EEG and ECG and the absence of reaction to noxious stimuli. This protocol was approved by the Yale University Institutional Animal Care and Use Committees and conforms to the guidelines recommended in Preparation and Maintenance of Higher Mammals during Neuroscience Experiments (National Institutes of Health publication 91-3207).

Recording. Intracellular and extracellular recordings were performed in area 17 , within $10^{\circ}$ of the area centralis representation. Intracellular recordings were obtained with sharp microelectrodes filled with $2 \mathrm{M} \mathrm{K}^{+}$acetate beveled to a final resistance of 50-100 $\mathrm{M} \Omega$. Intracellular recordings were included if the neuron showed a stable membrane potential negative to $-55 \mathrm{mV}$ at rest, showed an input resistance larger than 20 $\mathrm{M} \Omega$, and was able to discharge continuously with injection of depolarizing current pulses. Extracellular recordings were obtained with tungstenin-glass microelectrodes (Merrill and Ainsworth, 1972), and single units were sorted with a commercially available spike-sorting algorithm (Spike2; Cambridge Electronics Design, Cambridge, UK).

The size, shape, length, eye dominance, and velocity preferences of discharge RFs for each neuron were first determined with a hand-held projector. Subsequently, visual stimuli were generated and presented through a VSG-Series 3 computer system (Cambridge Research Systems, Cambridge, UK) on a 19-inch color monitor ( $80 \mathrm{~Hz}$ non-interlaced refresh; $1024 \times 768$ resolution). All visual stimuli were delivered through the dominant eye only. Quantitative determination of preferred orientation and spatial frequency was obtained from peristimulus time histograms (PSTHs) calculated on-line.

Sparse noise stimulation technique. A sparse noise stimulation technique was used for the quantitative mapping of RFs (Stevens and Gerstein, 1976; Jones and Palmer, 1987a; DeAngelis et al. 1993). Bars of two different polarities (bright or dark) were randomly flashed one at a time at 16 different $x$-positions (making 32 stimuli total) for 50 or $62.5 \mathrm{~ms}$ along an axis perpendicular to the preferred orientation of the cell. The onset of each bar was coincident with the offset of the previously flashed bar. The screen background was kept at a luminance halfway between that of the white and black bars, and the contrast of the flashed bars was $80 \%$. In protocols including scotoma presentation, the bar length was equal to the height of the scotoma. Otherwise, the bar length was $10^{\circ}$ unless indication of end-zone inhibition had been observed during the hand mapping. The bar width was chosen to be one-fourth to oneseventh of the spatial period corresponding to the preferred spatial frequency of the cell. The spacing between adjacent $x$-positions was equal to one bar width.

RF maps calculation. The spike response was converted to a spike density function (an analog signal) by replacing each spike by a raised cosine bell (10 ms width). For the membrane potential data, the spikes were removed using an interpolation technique. So that the same analysis technique could be applied to the spike and synaptic responses, the spikeremoved membrane potential and spike density function were both calculated with a bin size of $5 \mathrm{~ms}$. For each stimulus position and polarity, an average of either the spike density function or the membrane potential was calculated over a period of -125 to $+225 \mathrm{~ms}$ with respect to stimulus onset. Each average was smoothed with a Gaussian filter (width of 3.6 $\mathrm{ms}$ ). Note that this smoothing was applied in the time domain; no smoothing was applied in the spatial domain. The 64 averages were collected into four separate two-dimensional (space vs time) matrices corresponding to the spike and synaptic responses to white and black bars. For simple cells, subtraction of the black bar matrices from the white bar matrices resulted in two additional matrices in which the responses to the white bar were increments and those to the black bar were decrements. The results are presented as space-time maps ( $x-t$ plots) (McLean and Palmer, 1989; DeAngelis et al., 1993) in which the RF is presented in three dimensions: time ( $x$-axis), space ( $y$-axis), and response strength ( $z$-axis). The amplitude of the largest noise value in the baseline $(-125$ to $0 \mathrm{~ms}$ relative to stimulus onset) was determined in each matrix. A response was considered to be significant if its amplitude was larger than 1.1 times the largest noise value in three consecutive bins. This approach allowed us to dismiss false positives regardless of the statistics underlying the noise distribution. This is a conservative approach, because, if the noise was distributed in a Gaussian manner, as often happened for the synaptic responses, then the $p$ value associated with our criteria would be extremely low $(\mathrm{p} \ll 0.01)$.

$R F$ fitting. The RF profile used for quantitative measurements of RF width and gain corresponded to the averaged response over $\pm 10 \mathrm{~ms}$ centered on the peak response in the $x$ - $t$ plots. The RF profiles of complex cells and those of simple cells with a single subfield were fit with a Gaussian, using the strongest of either the white or black bar RF profiles: $y=y_{0}$ $+A \times \exp \left(-\left(x-x_{c}\right)^{2} / 2 \sigma^{2}\right)$, where $y_{0}$ is the offset, $A$ is the gain of the RF (or the amplitude of the response) in spikes per second or in millivolts, $x_{c}$ is the center of the RF, and $\sigma$ is its SD ( $\approx$ half-width at $61 \%$ of peak height) in degrees.

The RF profiles of simple cells with more than one subfield were fit using a Gabor function, corresponding to a sinusoid modulated by a Gaussian envelope. The Gabor has been shown to be an optimal expression to formalize the RF features of simple cells (Field and Tolhurst, 1986; Jones and Palmer, 1987b): $y=A \times \exp \left(-\left(x-x_{c}\right)^{2} / 2 \sigma^{2}\right) \times \cos ((2 \times$ $\left.\left.\pi \times F_{\text {opt }} \times\left(x-x_{c}\right)\right)+\varphi\right)$, where, in addition to the parameters $A, x_{c}$, and $\sigma$ corresponding to the Gaussian envelope, $F_{\text {opt }}$ is the frequency of the $\mathrm{RF}$, and $\varphi$ is its phase. No offset $\left(y_{0}\right)$ is used in this formula because the offset is zeroed by the subtraction of the dark bar response matrix from the bright bar response matrix. For simple cells, the RF width was taken as the $\sigma$ of the Gaussian envelope. The fitting was performed using the nonlinear curve-fitting tools of Origin software (Microcal Software, Northampton, MA) and is based on a nonlinear least-squares minimization method. The goodness-of-fit was provided by an $r^{2}$ value. Fits for which the $r^{2}$ value was $<0.65$ have not been included. The $r^{2}$ for all fits had a median value of 0.944 for the discharge RFs $(n=120)$ and 0.935 for the synaptic RFs $(n=96)$. The $r^{2}$ was $<0.8$ in only 1.67 and $4.18 \%$ of the cases for discharge and synaptic RFs, respectively. 
Our measures of synaptic RFs were based on the membrane potential deviations near firing threshold caused by visually evoked synaptic responses. These changes in membrane potential (hyperpolarization and depolarization) correspond generally with the integration field of inhibitory and excitatory conductances, although differences may exist between the conductance and its effect on membrane potential at rest (Monier et al., 2003). Therefore, our term "synaptic RF" corresponds to the cumulative effect of synaptic potentials at normal membrane potentials only. Deconvolving the synaptic integration field into its individual excitatory and inhibitory conductance components and then examining the effects of adaptation to highcontrast, low-contrast, and scotoma presentations on these individual components is beyond the scope of the present study. Here, we use the visually evoked membrane potential deviations as a first approximation of the synaptic RF.

An SE value was provided with each parameter of the fit. This allowed us to determine the significance of the effects of a given manipulation for each cell and for each parameter individually. A given parameter was considered to be significantly (at a $p$ level of 0.05 ) affected when its value \pm 1 SE in one condition did not overlap with the value $\pm 1 \mathrm{SE}$ obtained in the other condition.

Visual stimulation protocols. We examined the effects of adaptation to three different visual patterns: gray screen, whole-field high-contrast sine-wave gratings, and artificial scotoma. The luminance for the gray screen $(0 \%$ contrast) was set at the value corresponding to the average luminance of the grating of the whole-field or scotoma stimulus. Maintaining the same mean luminance does not make the artificial scotoma analogous to the effects of localized lesions in the retina, but this has the advantage that it should not affect the tonic activity of thalamic neurons, thus suppressing one variable that could have affected the results. The wholefield stimulus consisted of a $40-80 \%$ (usually $40 \%$ ) contrast sine-wave grating over the entire monitor, with the orientation, spatial frequency, and drift velocity being optimal for the recorded cell. The artificial scotoma was a gray square of $6.6 \pm 1.6^{\circ}$ side (mean $\pm \mathrm{SD}$; range of $4-9^{\circ}$ ) centered over the discharge RF and covering the same stimulus as used for the wholefield grating. The size of the scotoma was adjusted until presentation of the peripheral grating did not elicit any audible spike discharge. Scotoma of this size are within the limits of the modulatory surround measured in cat visual cortex (DeAngelis et al., 1994; Li and Li, 1994; Sengpiel et al., 1997; Palmer and Nafziger, 2002). Sequences of two to four of these visual patterns were presented for 15-30 s each in a looped manner to allow for averaging. In addition, the interleaved presentation of stimuli minimized the influence of firing variability over time. In most experiments, the RF mapping data were collected for 2.5 or $5 \mathrm{~s}$ in between presentation of the three visual patterns. For example, in Figures 1 and 2, the aftereffects of all three stimuli were examined in a sequence of gray mapping, scotoma mapping, high-contrast mapping. In Figure 4 , this sequence also included an additional scotoma presentation after the high-contrast stimulus and the mapping stimuli were removed so that simple cell.

\section{Simple cell, extracellular recording}
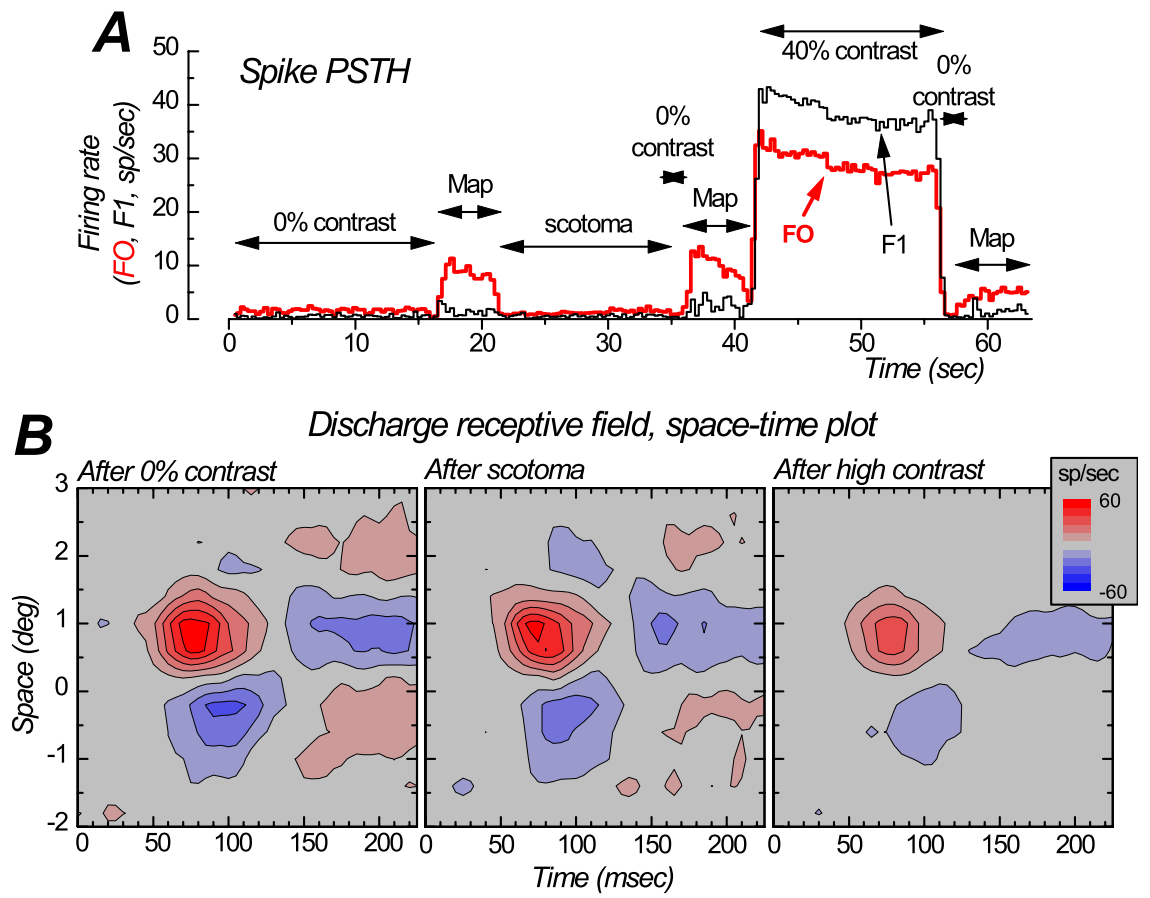

C Discharge receptive field profile through peak response

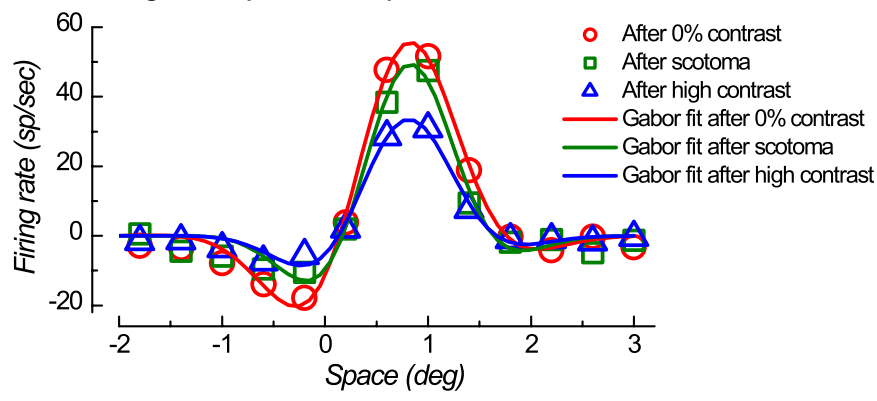

Figure 1. Effects of high-contrast and artificial scotoma on the discharge RF in a cortical simple cell. $A$, Mean firing rate $\left(F_{0}\right.$ component, in red) and the discharge that is modulated at the temporal frequency of the grating drift ( $F_{1}$ component, in black) during the presentation of a gray screen, scotoma ( $40 \%$ contrast, except for a $0 \%$ contrast square $6^{\circ}$ on a side centered over the $\mathrm{RF}$ ), and finally a whole-screen, $40 \%$ contrast grating. In between each of these stimuli, the RF was mapped for $5 \mathrm{~s}$ as indicated. The entire sequence was repeated in a loop 69 times for averaging. $B$, Discharge RF maps obtained after each of the three stimulus conditions in $A$. The $x$-axis is time, the $y$-axis is space, and the $z$-axis, color coded, represents the firing rate as a function of both space and time. Each map has been obtained after subtraction of the map obtained in response to dark bar stimuli to the map obtained in response to bright bar stimuli. Increase in red saturation represents increased response to the bright bar, and increase in blue saturation represents increased response to dark bar. Between $\sim 50$ and $\sim 130 \mathrm{~ms}$, the $x-t$ map shows two well defined subfields, corresponding to the $0 \mathrm{~N}$ response to the bright and $0 \mathrm{~N}$ response to the dark stimuli (in red and blue, respectively). After $\sim 130 \mathrm{~ms}$, the response polarity is reversed. This reversion occurs after the end of the stimulus and corresponds to the OFF response to the bright bars (in effect, a contrast decrement from the brightness of the bar to the gray screen, equivalent to a dark bar response, thus appearing in blue) and OFF response to the dark bars (contrast increment from the dark stimulus to the gray screen, equivalent to a bright bar response, thus appearing in red). C, RF profile at time of peak response and Gabor fits of the data obtained for the three stimulus conditions. The data points represent experimental measures, and the lines represent the best fit through these data. The fits reveal a significant decrease in gain after high-contrast stimulation, to a value representing $58 \%$ of the gain obtained after $0 \%$ contrast. Change in RF width ( $90 \%$ ) was not significant. Scotoma presentation significantly reduced RF gain (84\%) but did not have a significant effect on RF width (88\%). This data were obtained from an extracellularly recorded

the amplitude-time course of membrane potential and action potential firing rate changes could be examined in detail. In Figures 8 and 9, the effects of adaptation to high-contrast stimuli on synaptic and discharge RFs were examined with a simpler protocol of gray mapping, high-contrast mapping. All of these sequences were repeated several times (mean $\pm \mathrm{SD}, 23 \pm 17$ ) in a looped manner. This had the consequence that the beginning of the gray screen presentation followed the mapping after adaptation to either a sco- 
Complex cell, extracellular recording

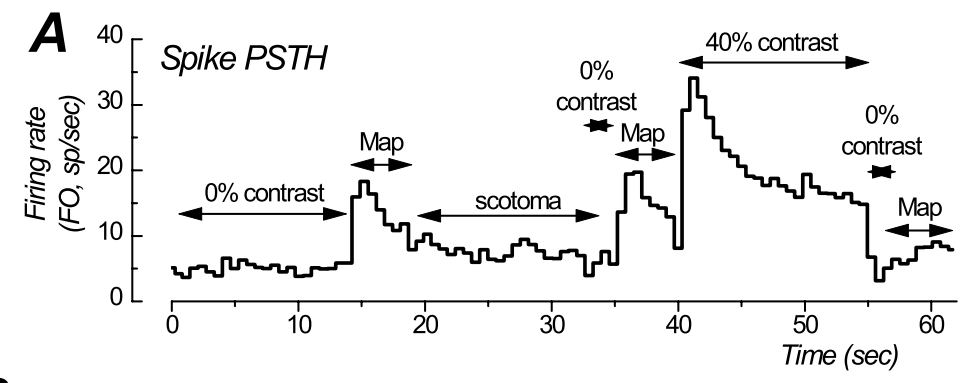

B

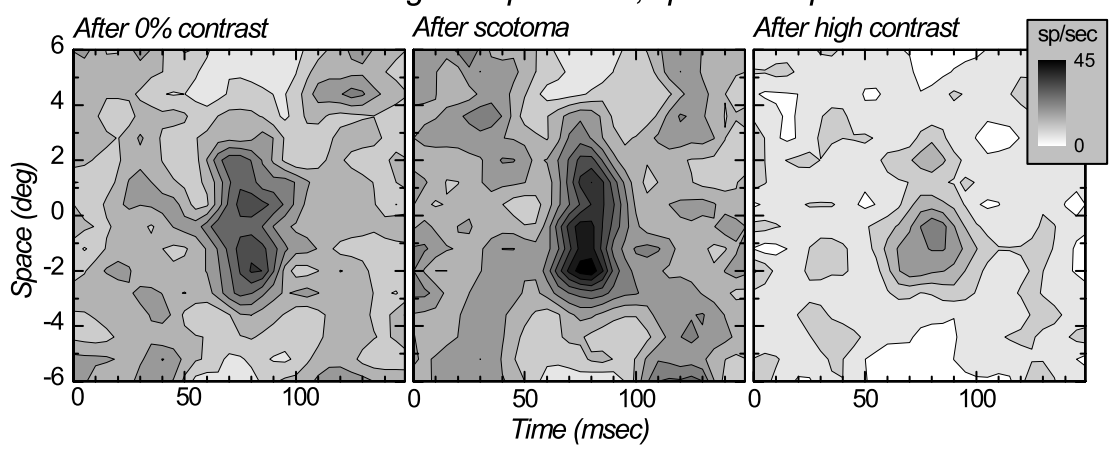

C Discharge receptive field profile through peak response

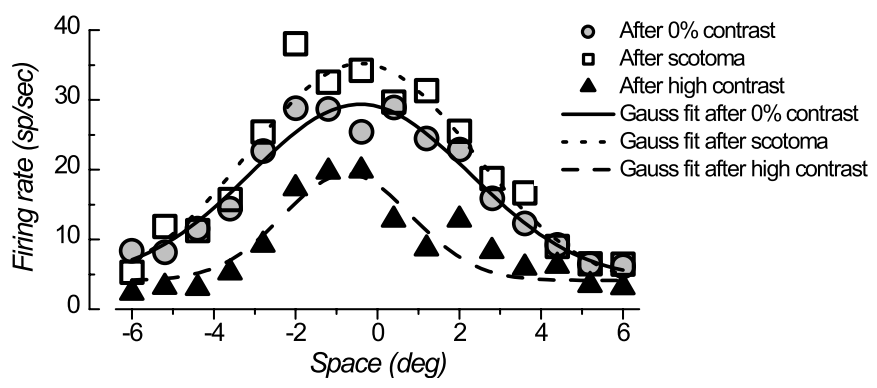

Figure 2. Effects of high-contrast and artificial scotoma on the discharge RF in a cortical complex cell. $A$, Discharge rate as a function of time plotted for the mean firing rate ( $F_{0}$ component). The sequence of stimuli, repeated 62 times, is labeled above the time series and follows the same order as in Figure 1 . Scotoma side, $6^{\circ}$. B, Discharge RF profiles as mapped with the dark bar stimuli: left, after $0 \%$ contrast; middle, after scotoma presentation; right, after high contrast. C, RF profiles (symbols) at peak response after the three stimulus conditions and corresponding Gaussian fits (lines). Comparison of parameters extracted from the fits indicates that high-contrast stimulation was followed by a significant reduction of both RF gain and width (to 61 and $59 \%$ of the gain and width values after $0 \%$ contrast stimulation, respectively). On the other hand, the scotoma presentation induced an increase in gain (to $129 \%$ of the value obtained after $0 \%$ contrast stimulation) but no significant change in RF width (103\%). These data were obtained from an extracellularly recorded complex cell.

toma or high-contrast stimulus. Baseline (control) receptive fields were taken as those after adaptation to a 15-20 s period of unpatterned stimulation (i.e., a gray screen).

To examine the influence of the intrinsic hyperpolarizing currents of the cells on RF width and gain, we activated these by intracellular injection of sinusoidal current $(10-20 \mathrm{~s}$ at $2 \mathrm{~Hz}, \sim 1 \mathrm{nA})$. To maintain the cell and its afferent inputs in a state of steady visual adaptation, the visual mapping stimulus was continuously presented. Finally, to examine the effect of passive hyperpolarization on RFs, we mapped the RF while polarizing the cell to different membrane potentials through the intracellular injection of constant current.

In protocols with grating stimuli (either whole-screen or scotoma) and sinusoidal current injection, the PSTHs for the spike responses and average membrane potential are displayed as a time series of the $F_{0}$ component (see Figs. 1, 2, 8, 9, 11). The $F_{1}$ component is also displayed for simple cells and for current injection protocols (see Figs. $1,8,11$ ). The $F_{0}$ and $F_{1}$ components were obtained after a Fourier transform of the PSTH or of the membrane potential average elicited by each cycle of the drifting grating (or of the sinusoidal current) with the same ordinal position across the several repeats of the stimuli (for details, see Sanchez-Vives et al., 2000a). A cell-by-cell statistical analysis of the significance of the membrane potential and firing rate changes induced by or after the presentation of the scotoma, the high-contrast grating, and the sinusoidal current injection was performed using a $t$ test, with baseline values deriving from the five consecutive $F_{0}$ points at the end of adaptation to a gray screen (or before current injection). Depending on the drift velocity of the grating, five values correspond to either 1.6 or $0.8 \mathrm{~s}$. The changes that were measured include the following (see Fig. 4; all measured as five consecutive $F_{0}$ values of the appropriate period): (1) membrane potential depolarization and changes in action potential firing rate with the beginning of high-contrast or scotoma stimulation; (2) degree of adaptation at the end of high-contrast or scotoma stimulation; (3) aftereffects of high-contrast adaptation measured using the first five values of either gray screen or scotoma stimulation that follow this adaptation; and (4) aftereffects of scotoma presentation measured using the beginning of the consecutive gray screen presentation. (Note that, in some experiments in which mapping of the RF was performed, such as those presented in Figs. 1, 2 , and 9, the aftereffects of scotoma or highcontrast stimulation were determined during a $1 \mathrm{~s}$ presentation of a gray screen that was inserted between the scotoma or the high-contrast stimulus and the mapping stimulus.)

Statistics. All population data in the text are given as mean \pm SD. Except for the cell-by-cell analysis described above, all statistical comparisons have been made using the nonparametric Wilcoxon paired test, and correlations between measured parameters were tested with the nonparametric Spearman's rank correlation test.

\section{Results}

One or several visual stimulation protocols designed to investigate the cellular mechanisms of RF dynamics were applied to 54 cortical neurons in area 17 (7 extracellular and 47 intracellular recordings). Electrophysiologically (Nowak et al., 2003), these cells were characterized as 28 regular-spiking cells, 11 chattering cells, 6 intrinsically bursting neurons, and 2 fastspiking cells. Seven cells either were not classified electrophysiologically or were intermediate between these classifications.

Cells of both the simple and complex RF subtypes were identified according to their responses to drifting sinusoidal gratings and/or one-dimensional RF maps. In one-dimensional RF maps (Hubel and Wiesel, 1962; Movshon et al., 1978a,b; Field and Tolhurst, 1986; Heggelund, 1986; Jones and Palmer, 1987a; DeAngelis et al., 1993), simple cells $(n=26)$ exhibited either a single dark- or a single bright-excitatory subfield ( $\mathrm{S} 1$ cells) or between two and four non-overlapping dark- and brightexcitatory subfields. Complex cells displayed dark- and brightexcitatory subfields that overlapped in space and time $(n=12)$. All four electrophysiological classes consisted of both simple and complex cells. In cases for which no RF map was available, simple $(n=5)$ and complex cells $(n=5)$ have been differentiated on the 
basis of the modulation of their spike responses to the presentation of a sine-ave drifting grating of optimal spatial and temporal frequency (Skottun et al., 1991). The visual response in six cells was too poor to allow for an identification of RF type; these cells have been kept in the sample because they provided data about the aftereffects of sinusoidal current injection on the membrane potential but were not used for visual response analysis.

\section{Adaptation to high-contrast stimuli results in a decrease in discharge RF gain and width, whereas adaptation to an artificial scotoma does not}

We first determined how the width and gain of discharge RFs are affected by previous adaptation (for 15-30 s) to a gray screen, a high-contrast grating (usually $40 \%$ contrast), or an artificial scotoma (average size of $6.6 \pm 1.6^{\circ}$ ) (Figs. 1-3). Presentation of a high-contrast stimulus increased firing rate well above spontaneous activity (Figs. $1 A, 2 A$ ). During the prolonged (15-30 s) presentation of the high-contrast stimulus, the discharge rate of both simple (Fig. 1) and complex (Fig. 2) cells progressively decreased, as reported previously (Maffei et al., 1973; Albrecht et al., 1984), constituting the phenomenon of contrast adaptation. After removal of the high-contrast stimulus, the firing rate typically fell below that occurring just before adaptation to the highcontrast stimulus (Figs. $1 \mathrm{~A}, 2 \mathrm{~A}$ ).

The gain of the discharge RFs after adaptation to the highcontrast stimulus was consistently reduced in both simple and complex cells (Figs. $1 B, C, 2 B, C$ ). At the population level, the gain was significantly $(p<0.0001)$ and strongly decreased (on average, to $56.1 \pm 29.0 \%$; range of $0-128 \% ; n=21$ cells including 5 extracellularly recorded cells) compared with that after adaptation to $0 \%$ contrast (Fig. $3 A$; Table 1). When examined on a cell-by-cell basis, this gain reduction was significant in 18 of 21 cells (Table 1).

High-contrast stimulation also resulted in a significant ( $p=$ 0.03 ) decrease in discharge RF width, with an average reduction to $88.4 \pm 19.5 \%$ when compared with the control value measured after $15-30$ s of $0 \%$ contrast stimulation $(n=19$ cells including 5 extracellular recordings) (Fig. 3B; Table 1). Width reduction was weaker than gain reduction and, when examined on a cell-by-cell basis, the weakness of the effect observed at the population level is confirmed, with only 4 of 19 cells showing a significant reduction of RF width (Fig. 2B, C shows one of these cases). There was no significant correlation between change in width and gain of the discharge RF (Fig. 3C); this indicates that cells showing large gain reduction did not necessarily show strong RF shrinkage. The relative weakness of discharge RF width reduction may lead to the argument that, compared with the strength of the discharge RF gain reduction, $\mathrm{RF}$ width is relatively invariant with respect to contrast adaptation.

In contrast to the aftereffects of high-contrast stimulation, presenting an artificial scotoma did not lead to statistically significant effects on either discharge RF gain (106.5 $\pm 22.1 \%$; $p=$ $0.25)$ or width $(95.6 \pm 15.8 \% ; p=0.5 ; n=16$ cells including 5 extracellular recordings) compared with the effects of adapting to a gray screen (Figs. 1, 2, 3D,E; Table 1). Therefore, the visual stimulation of the RF surround does not have an obvious effect on the subsequently measured discharge RF.

\section{Adaptation to high-contrast stimuli is associated with a membrane potential hyperpolarization, whereas adaptation to a scotoma does not have strong effects on the membrane potential}

Intracellular recordings allowed us to examine changes in both firing rate and average membrane potential during 15-30 s of adaptation to gray screen, scotoma, and high-contrast stimuli (Fig. $4 A, B$ ). The presentation of a high-contrast stimulus resulted in an increase in firing rate from a mean spontaneous activity level of $4.3 \pm 4.0 \mathrm{~Hz}$ to a mean rate of $35.3 \pm 28.1 \mathrm{~Hz}(n=$ 28 intracellular and 7 extracellular recordings) (Fig. 4C). The firing rate then adapted so that, at the end of the high-contrast stimulation, it averaged $18.3 \pm 15.6 \mathrm{~Hz}$ (Fig. 4C). The membrane potential ( $F_{0}$ component) paralleled these changes in firing rate, increasing by $4.3 \pm 2.6 \mathrm{mV}(n=28)$ at the beginning of highcontrast stimulation and then reaching a value of $1.7 \pm 2.5 \mathrm{mV}$ above the $0 \%$ contrast value at the end of high-contrast stimulation (Fig. 4D).

The aftereffects of high-contrast stimulation were examined in 17 intracellularly recorded neurons in which either an artificial scotoma $(n=5)$ or a gray screen $(n=12)$ followed adaptation to the high-contrast whole-screen stimulus (11 cells were not considered for this analysis because the stimulus immediately after the high contrast was the sequence of flashed bars used for mapping the RF). As in Figure $4 B$, presentation of either an artificial scotoma or a gray screen after high-contrast stimulation was associated with, on average, a hyperpolarization of the membrane potential of $3.4 \pm 2.9 \mathrm{mV}(p=0.0003)$ (Fig. $4 D)$. When examined on a cell-by-cell basis (see Materials and Methods), 14 cells exhibited a significant hyperpolarization, and three cells showed no significant membrane potential change. This hyperpolariza- 
Table 1. Effects of different manipulations on synaptic and discharge RFs width and gain

\begin{tabular}{|c|c|c|c|c|c|}
\hline \multirow[b]{2}{*}{ Protocol } & \multicolumn{2}{|l|}{ Discharge RF } & \multicolumn{2}{|l|}{ Synaptic RF } & \multirow[b]{2}{*}{$\begin{array}{l}\text { Membrane potential change } \\
(\mathrm{mV})\end{array}$} \\
\hline & $\begin{array}{l}\text { Gain change } \\
(\%)\end{array}$ & $\begin{array}{l}\text { Width } \\
\text { change } \\
(\%)\end{array}$ & $\begin{array}{l}\text { Gain change } \\
(\%)\end{array}$ & $\begin{array}{l}\text { Width change } \\
\text { (\%) }\end{array}$ & \\
\hline Aftereffects of high-contrast stimulation & $\begin{array}{l}56.1 \pm 29.0 \\
n=21 \\
\text { Sig. Inc. }=0 \\
\text { Sig. Dec. }=18\end{array}$ & $\begin{array}{l}88.4 \pm 19.5 \\
n=19 \\
\text { Sig. Inc. }=1 \\
\text { Sig. Dec. }=4\end{array}$ & $\begin{array}{l}88.3 \pm 42.9 \\
n=15 \\
\text { Sig. Inc. }=2 \\
\text { Sig. Dec. }=4\end{array}$ & $\begin{array}{l}87.6 \pm 20.6 \\
n=13 \\
\text { Sig. Inc. }=1 \\
\text { Sig. Dec. }=3\end{array}$ & $\begin{array}{l}-1.97 \pm 1.20 \\
n=15\end{array}$ \\
\hline Aftereffects of scotoma stimulation & $\begin{array}{l}106.5 \pm 22.1 \\
n=16 \\
\text { Sig. Inc. }=3 \\
\text { Sig. Dec. }=2\end{array}$ & $\begin{array}{l}95.6 \pm 15.8 \\
n=16 \\
\text { Sig. Inc. }=0 \\
\text { Sig. Dec. }=2\end{array}$ & $\begin{array}{l}101.9 \pm 13.8 \\
n=10 \\
\text { Sig. Inc. }=1 \\
\text { Sig. Dec. }=0\end{array}$ & $\begin{array}{l}101.6 \pm 15.7 \\
n=10 \\
\text { Sig. Inc. }=0 \\
\text { Sig. Dec. }=0\end{array}$ & $\begin{array}{l}-0.10 \pm 0.46 \\
n=10\end{array}$ \\
\hline Mapping at different membrane potentials & $\begin{array}{l}71.9 \pm 18.7 \\
n=13 \\
\text { Sig. Inc. }=0 \\
\text { Sig. Dec. }=9\end{array}$ & $\begin{array}{l}81.7 \pm 14.5 \\
n=13 \\
\text { Sig. Inc. }=0 \\
\text { Sig. Dec. }=9\end{array}$ & $\begin{array}{l}130.0 \pm 24.7 \\
n=13 \\
\text { Sig. Inc. }=10 \\
\text { Sig. Dec. }=0\end{array}$ & $\begin{array}{l}95.2 \pm 15.0 \\
n=13 \\
\text { Sig. Inc. }=1 \\
\text { Sig. Dec. }=3\end{array}$ & $\begin{array}{l}-3.65 \pm 1.85 \\
n=13\end{array}$ \\
\hline Aftereffects of sinusoidal current injection & $\begin{array}{l}82.5 \pm 22.0 \\
n=10 \\
\text { Sig. Inc. }=0 \\
\text { Sig. Dec. }=4\end{array}$ & $\begin{array}{l}93.8 \pm 13.9 \\
n=10 \\
\text { Sig. Inc. }=0 \\
\text { Sig. Dec. }=2\end{array}$ & $\begin{array}{l}107.5 \pm 21.4 \\
n=10 \\
\text { Sig. Inc. }=3 \\
\text { Sig. Dec. }=1\end{array}$ & $\begin{array}{l}102.9 \pm 1.5 \\
n=10 \\
\text { Sig. Inc. }=1 \\
\text { Sig. Dec. }=0\end{array}$ & $\begin{array}{l}-1.55 \pm 1.85 \\
n=10\end{array}$ \\
\hline
\end{tabular}

Changes in gain and width for both synaptic and discharge RFs are given as percentage relative to the following: (1) the gain and width of the RF measured after 15-30 s of unpatterned visual stimulation (gray screen) for high-contrast and scotoma protocols; (2) the RF measured without hyperpolarizing current (or with lower amount of injected current) in the "mapping at different membrane potential" protocol; and (3) the RF measured before current injection in the "sinusoidal current injection" protocol. Changes in membrane potential correspond to the mean membrane potential during mapping after the manipulation subtracted from the mean membrane potential during mapping before the manipulation. Values given correspond to the mean \pm SD; $n$ refers to the sample size. Sig. Inc., Significant increase; Sig. Dec., significant decrease. Numbers given correspond to the number of cells that showed a significant increase or a significant decrease for the parameter under consideration in a cell-by-cell analysis.

tion was associated with a decrease of the spontaneous firing rate to $2.8 \pm 3.0 \mathrm{~Hz}(p=0.04)$ (Fig. $4 C)$.

The time constant of recovery from the hyperpolarization was on average $6.7 \pm 4.8 \mathrm{~s}$ and ranged from 1.6 to $15.1 \mathrm{~s}$ (data not illustrated; $n=8$; fit with a single-exponential curve and including only cells in which the $r^{2}$ of the fit to the data were $>0.5$ ). These effects of high-contrast stimulation are similar to those shown previously in studies of contrast adaptation in cortical neurons (Carandini and Ferster, 1997; Sanchez-Vives et al., 2000a) in which adaptation to a high-contrast stimulus, and adaptation aftereffects, occur in part through a hyperpolarization of the membrane potential.

Presentation of an artificial scotoma caused a small, but statistically significant increase in spike rate to $6.2 \pm 4.5 \mathrm{~Hz}$ compared with the spontaneous rate of $3.3 \pm 3.0 \mathrm{~Hz}(p=0.0002 ; n=$ 28 ) at the later portions of adaptation to a gray screen (Fig. $4 E$ ). At the end of the scotoma presentation, the firing rate was still elevated $(4.2 \pm 2.9 \mathrm{~Hz} ; p=0.03)$ but by $<1 \mathrm{~Hz}$ on average compared with the mean spontaneous activity level during gray screen presentation (Fig. $4 E$ ).

At the membrane potential level, a small depolarization paralleled, on average, the weak firing rate increase observed at the beginning of the scotoma presentation $(+1.2 \pm 2.0 \mathrm{mV} ; n=21$; $p=0.003$ ) (Fig. $4 F)$. Statistical analysis on a cell-by-cell basis revealed a diversity of effects: there was either a depolarization $(n=10 ;+2.5 \pm 1.5 \mathrm{mV})$ or no significant changes $(n=9$; $+0.6 \pm 0.8 \mathrm{mV})$, or the cell exhibited a hyperpolarization $(n=2$; $-2.6 \pm 2.2 \mathrm{mV}$ ). These membrane potential changes adapted during the 15-30 s of scotoma presentation such that, by the end of the scotoma period, the membrane potential value was not significantly different from before the presentation of the artificial scotoma $(p=0.26$; mean difference, $+0.3 \pm 1.2 \mathrm{mV}$ ) (Fig. $4 F)$.

Finally, we examined whether prolonged scotoma stimulation resulted in aftereffects on the membrane potential. We found that the membrane potential after presentation of the artificial scotoma was not significantly different from that occurring after the presentation of a gray screen (mean \pm SD difference, $-0.12 \pm 1.0$ $\mathrm{mV} ; n=21 ; p=0.3$ ) (Fig. $4 F$ ).
In conclusion, our results indicate that adaptation to an artificial scotoma does not produce any strong or long-lasting effects on membrane potential compared with the membrane potential changes associated with adaptation to a gray screen. The main effect observed here is that presentation of a high-contrast stimulus depolarizes visual cortical neurons; this depolarization, and the associated spike discharge, lessens during adaptation. After replacement of the high-contrast stimulus with either a scotoma or a gray screen, there is a significant membrane potential hyperpolarization, whose amplitude lessens during adaptation to the scotoma or gray screen, resulting in a slow restoration of neuronal excitability.

\section{Iceberg effect: hyperpolarization reduces discharge RF gain and width but leaves synaptic RF width unaffected and increasing synaptic RF gain}

As shown above, presentation and withdrawal of a strong visual stimulation is followed by a hyperpolarization that slowly dissipates, thereby causing slow membrane potential changes of several millivolts. We hypothesized that these membrane potential fluctuations have significant effects on both the size and gain of discharge RFs through a simple iceberg effect. Here, we directly tested this possibility through maintained changes in the membrane potential produced with the intracellular injection of DC while mapping the discharge RF (Figs. 5-7).

Hyperpolarizing the membrane potential with the intracellular injection of DC by an average of $3.6 \pm 1.8 \mathrm{mV}(n=13)$ resulted in a significant $(p=0.003)$ reduction of the discharge RF gain to $71.9 \pm 18.7 \%$ (control vs hyperpolarized) (Figs. $5 A, C$, $7 B$ ) and a significant $(p=0.003)$ decrease in discharge RF width to $81.7 \pm 14.5 \%$ of the depolarized value (Figs. $5 C, 6 C, 7 E$ ). When examined on a cell-by-cell basis, hyperpolarization resulted in a significant decrease in both the gain and width of discharge RF in 9 of 13 cells, whereas in four cells, there was no significant change (Table 1).

As could be expected with DC injection, the synaptic RF did not show significant changes in width with hyperpolarization $(95.2 \pm 15.0 \% ; p=0.09)$ (Figs. $5 D, 6 D, 7 D)$. However, there was 

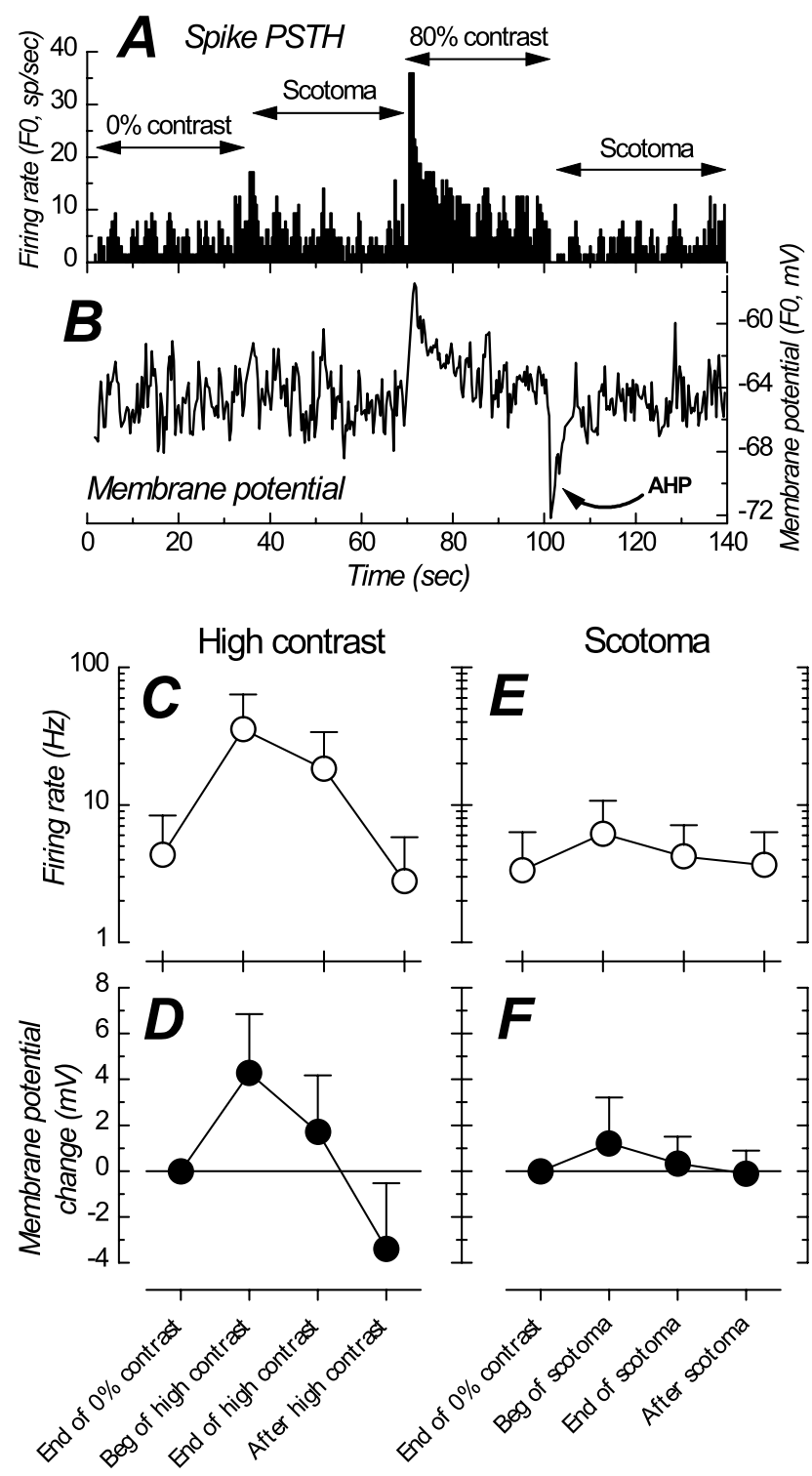

Figure 4. Average membrane potential of cortical neurons depends on temporal and spatial features of visual stimulation. $A$, PSTH of spike response ( $F_{0}$ component time series) for an intracellularly recorded complex cell in a four-stimulus protocol without RF mapping, as indicated. The scotoma was a $5^{\circ}$ square, and the entire sequence was repeated four times for averaging. $B$, Membrane potential average ( $F_{0}$ component time series) during the stimulation protocol of $A$. $C-F$, Population summary (mean $+S D$ ) of the effects and aftereffects of highcontrast or scotoma stimulation on firing rate $(C, E)$ and membrane potential $(D, F)$. Each point represents the average of five $F_{0}$ values, corresponding to either 0.8 or $1.6 \mathrm{~s}$ (depending on grating drift temporal frequency). The "After high contrast" point is the average of the first five points of either the $0 \%$ contrast or scotoma, depending on whichever followed the highcontrast stimulus. In some experiments in which mapping of the RF was performed (Figs. 1, 2,9), the "After high contrast" values were determined from the second of gray screen that was inserted between the high-contrast stimulus and the mapping stimulus. In $E$ and $F$, "After scotoma" corresponds to the value at the beginning of the gray screen presentation with some exceptions. (1) in some experiments in which mapping of the RF was performed (Figs. 1, 2), the "After scotoma" value was determined from the second of gray screen that was inserted between the scotoma and the mapping stimulus. (2) In some experiments, no gray screen was inserted between the end of the scotoma and the RF mapping. For these cells, the baseline corresponds to the mean values at the beginning of the mapping after $0 \%$ contrast, and the "After scotoma" value was determined from first values during the mapping after scotoma. Membrane potential changes in $D$ and $F$ are expressed relative to the membrane potential at the end of the $0 \%$ contrast stimulation. The spontaneous activity level is different in $C$ and $E$ because the cell samples are not the same.
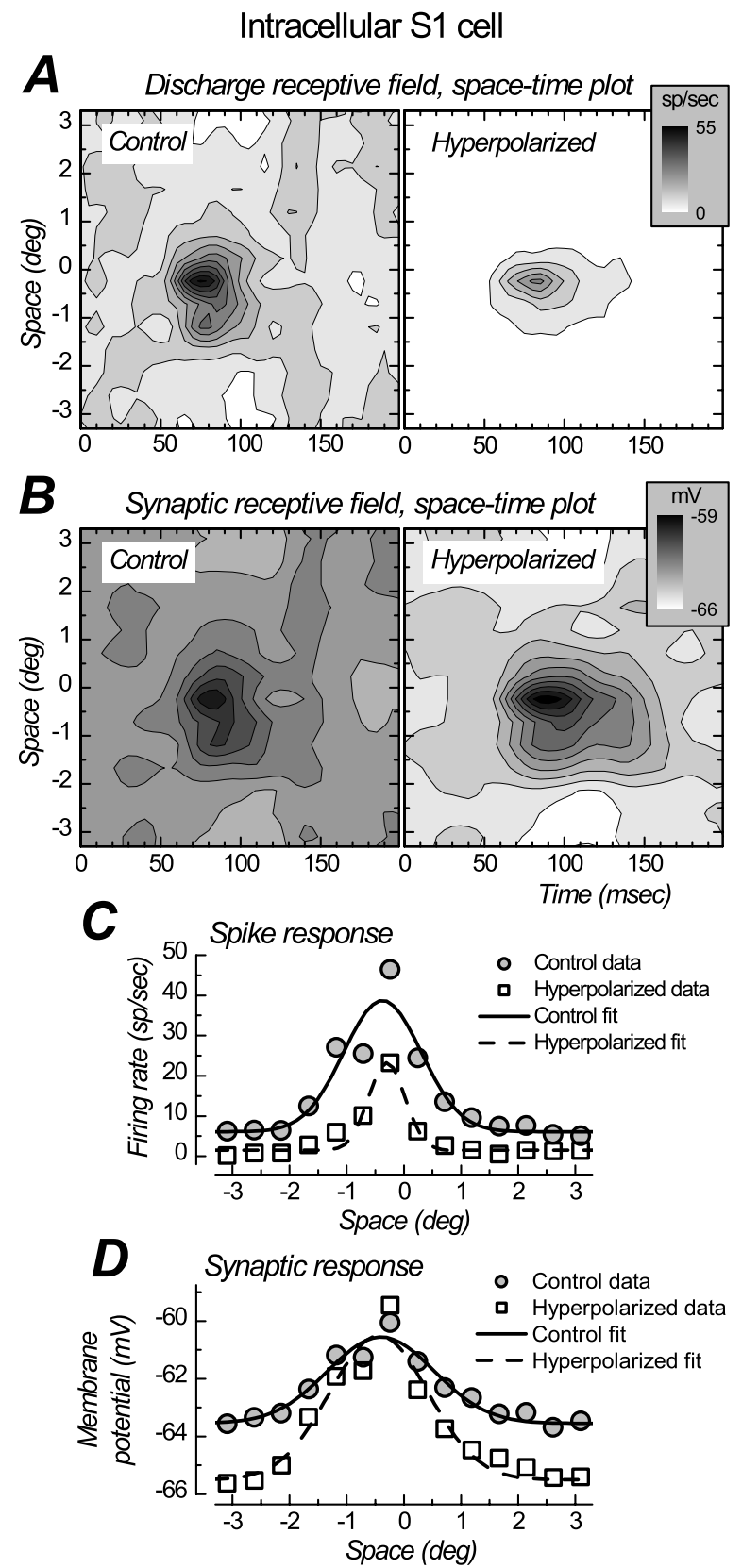

Figure 5. Effects of hyperpolarization of the membrane potential on the discharge and synaptic RF in a simple $(S 1)$ cell. $A$, Space versus time $(x-t)$ plots of the discharge RF of a simple cell with a single subfield (activated by dark bars only). The RF map on the right (hyperpolarized) was obtained while the cell was hyperpolarized by $3 \mathrm{mV}$ with the intracellular injection of current with respect to the map on the left. Note decreased spontaneous activity and decreased response strength. $B$, Space versus time plots of the synaptic RF obtained in the same conditions. C, Spatial RF profiles made for the time of the peak response in the discharge RF map. Gaussian curves have been fitted to the experimental data points. The gain ( $67 \%$ of control) and width (47\%) of the discharge RF were significantly reduced when the cell was hyperpolarized. $D$, Spatial RF profiles made for the time of the peak response in the synaptic RF map. No significant change in width (92\%) was observed, but the hyperpolarization induced an increase in gain (167\%) in the synaptic RF.

a significant increase (by $30.0 \pm 24.7 \%$ ) in synaptic RF gain when cells were hyperpolarized ( $p=0.004$; statistically significant in 10 of 13 cells tested) (Figs. 5D, 7A; Table 1). This increase may have resulted from an increased driving force for excitatory synaptic potentials or from a reduction of the shunt produced by 


\section{Intracellular simple cell}

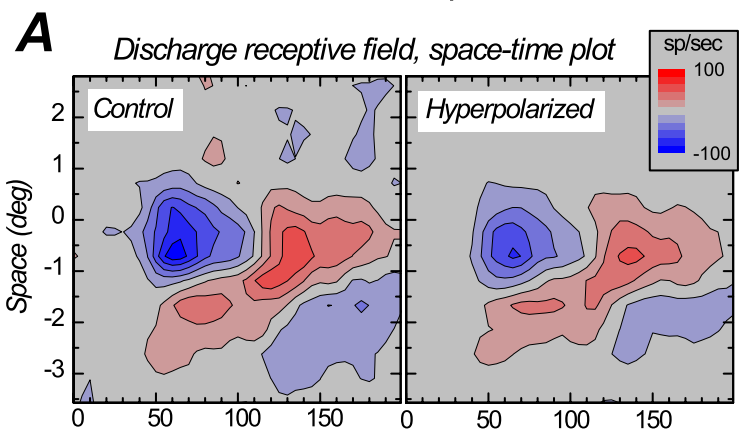

B Synaptic receptive field, space-time plot
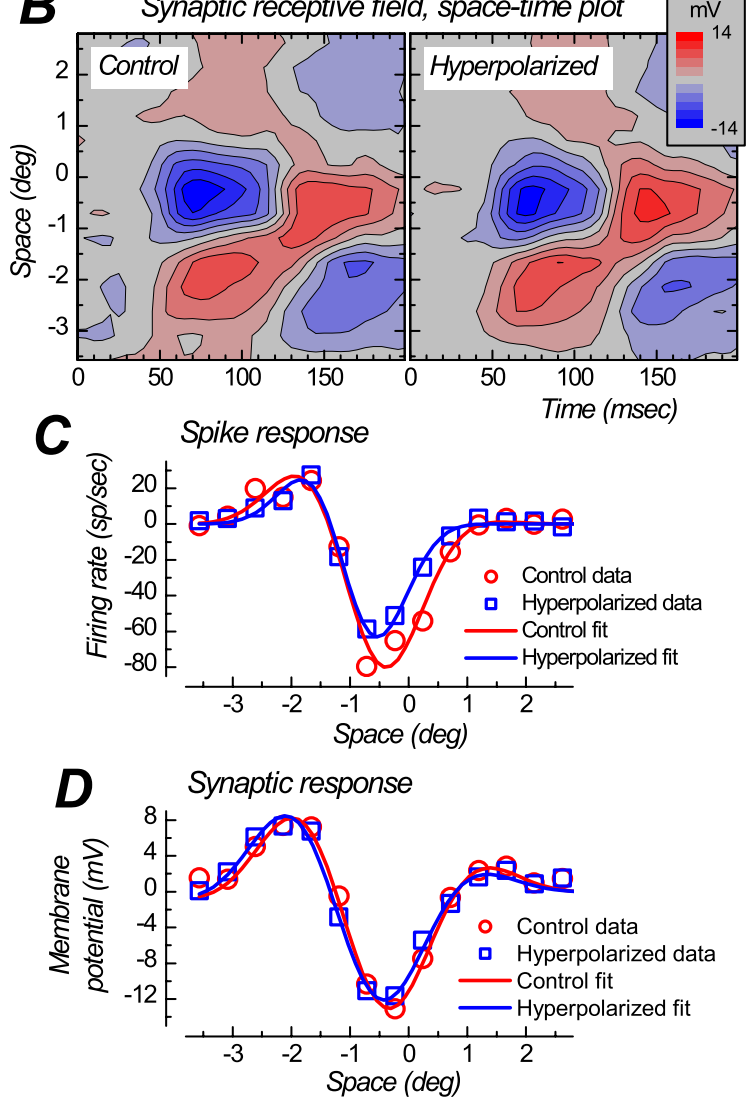

Figure 6. Effects of hyperpolarization of the membrane potential on the discharge and synaptic RF in a simple cell. $A, B$, Space versus time plots of the discharge and synaptic RF. Maps obtained after subtraction of dark bars response from bright bars response. The maps on the right were obtained while the cell was hyperpolarized by $2.2 \mathrm{mV}$ with $\mathrm{DC}$ compared with the maps on the left. C, Gabor fits of discharge RF profiles around peak response show weakly decreased gain (87\%) and significantly reduced width (84\%). D, Gabor fits of the synaptic RF profile do not show significant changes in gain (97\%) or width (99.5\%).

conductances associated with action potential firing, whose rate decreased with hyperpolarization.

\section{Both membrane potential hyperpolarization and synaptic receptive field shrinkage may contribute to $\mathrm{RF}$ dynamics after high-contrast stimulation}

So far, we demonstrated that the discharge RF gain and width as well as the membrane potential are dependent on the adaptation state of the neuron. Thus, after adaptation to a high-contrast stimulus, the gain, and sometimes the width, of the discharge RF is reduced, whereas the membrane potential is relatively hyperpolarized. We have shown that DC-induced hyperpolarizations also result in significant decreases in discharge RF gain, with smaller decreases in discharge RF width.

However, decreases in discharge RF gain and width after highcontrast stimulation may also occur through decreases in the synaptic inputs arriving in the neuron. To examine this possibility, we directly compared, in the same neurons, the changes in discharge RFs with that of synaptic RFs induced by adaptation to either a whole-field high-contrast stimulus or a gray screen (Figs. 8-10).

The firing rate, membrane potential, and discharge and synaptic RF maps are illustrated for a simple cell in Figure 8 and for a complex cell in Figure 9. Both of these cells exhibited significant decreases in discharge RF gain after adaptation to a high-contrast visual stimulus compared with those obtained after adaptation to a gray screen (Figs. $8 C, E, 9 C, E$ ). The discharge RF width was also significantly decreased in the complex cell (Fig. 9C,E). In addition, these effects were associated with the typical hyperpolarizations that follow adaptation to the high-contrast stimulus (Figs. $8 B, 9 B)$.

At the population level (Fig. $10 \mathrm{~A}$ ), the membrane potential during the mapping after the high-contrast stimulation was more hyperpolarized, by an average of $-1.97 \pm 1.20 \mathrm{mV}$ (range, -4.6 to $-0.3 \mathrm{mV} ; n=15$ ) compared with the membrane potential during the mapping after $0 \%$ contrast stimulation ( $p=0.0007)$.

In examining the effects of adaptation on synaptic RFs, we found variable, but for some cells, statistically significant effects. For example, the synaptic RF of the cell in Figure 8 showed no significant change in gain $(108 \%)$ and no significant change in width $(102 \%)$ (Fig. $8 D, F)$. In contrast, the synaptic RF of the complex cell in Figure 9 showed a significant decrease in width $(69 \%)$, although there was also a concomitant increase in synaptic RF gain (167\%) (Fig. 9D,F).

At the population level, the gain of the synaptic RF (Fig. 10B) was, on average, not modified ( $p=0.7$ ) after high-contrast stimulation. Expressed as a percentage, the change in synaptic RF gain averaged $88.3 \pm 42.9 \%$ (range, $0-166.7 \%$ ). However, this trend at the population level masks a variety of changes in individual cells: four of the 15 cells showed a significant reduction of gain after high-contrast stimulation (Table 1), including two cells for which the synaptic RF became undetectable, meaning that, after adaptation to a high-contrast stimulus, the mapping stimuli failed to evoke detectable synaptic responses (Fig. $10 \mathrm{~B}$, change in gain of $0 \%)$. Two cells showed a significant increase in synaptic RF gain (Table 1).

A close examination of the data suggests that both membrane potential and synaptic RF gain changes may contribute to the discharge RF gain reduction after high contrast (Fig. 10C,D). For example, one of the two complex cells for which the discharge RF gain was reduced to $0 \%$ showed a large membrane potential hyperpolarization with a minimal decrease in synaptic RF gain, whereas the other showed the exact opposite: that is, a weak membrane potential change but a complete suppression of the synaptic response.

One complicating factor in the interpretation of changes in synaptic RF gain after high-contrast stimulation is our finding that hyperpolarization with DC typically results in an increase in synaptic RF gain (Fig. 7A). Thus, the lack of an increase in synaptic gain during the high-contrast stimulation-induced hyperpolarization may actually represent a significant reduction of the synaptic RF gain. Because we did not correct for this, it is likely that the number of cells showing a significantly decreased synaptic RF gain is underestimated. 
In contrast to the synaptic RF gain, the synaptic RF width was significantly ( $p=$ 0.02 ) reduced after high-contrast stimulation (Fig. $10 F)$, the reduction averaging $87.6 \pm 20.6 \%$ of the value obtained after $0 \%$ contrast stimulation (range, 38.9$130.9 \% ; n=13$; two cells for which the synaptic RF was completely suppressed after high contrast could not be used for the calculation of changes in synaptic RF width). On a cell-by-cell basis, the reduction was significant in three cells, one cell showed a significant increase, and nine cells no significant change (Table 1 ).

There was no significant correlation between any of the following pairs of variables: amount of hyperpolarization and change in discharge RF width (Fig. 10G) and change in synaptic RF width and change in discharge RF width (Fig. $10 \mathrm{H}$ ). It is interesting to note that, in simple cells, synaptic RF width changes took values lower than $100 \%$ in the majority of cells, whereas the changes for the discharge RF were more scattered (Fig. $10 \mathrm{H}$ ). This suggests that high-contrast stimulation resulted in the suppression of synaptic subfields that were subthreshold for spike generation.

Altogether, these data indicate that the aftereffects of high-contrast adaptation on the discharge RF gain may be determined by both the membrane potential and synaptic RF gain modification. The decrease in discharge RF width after high contrast may result from a synaptic RF width reduction, but membrane potential hyperpolarization may contribute as well through an iceberg effect (compare with Fig. 7).

\section{Artificial scotoma, when presented after adaptation to $0 \%$ contrast, induces neither membrane potential nor RF changes}

We have shown (Figs. 1-3) that presenting an artificial scotoma for 15-30 s does not seem to have more effects on the discharge RF than presenting a full screen, $0 \%$ contrast stimulus. Furthermore, artificial scotoma presentation is not followed by significant aftereffects on the membrane potential changes. However, scotoma presentation (activation of the RF surround) may have had some subtle effects on the RF in its area that is subthreshold for spike generation. Examining the synaptic RF after adaptation to the artificial scotoma could reveal such a subtle effect.

No significant changes could be detected on the $x-t$ RF maps for the spike response and for the synaptic response after adaptation to an artificial scotoma (data not shown). At the population level (Table 1), the membrane potential during the mapping after scotoma was not different $(p=0.6)$ from the membrane potential during the mapping after $0 \%$ contrast stimulation (data not illustrated; mean difference, $-0.10 \pm 0.46 \mathrm{mV}$ ).

Furthermore, adaptation to an artificial scotoma resulted in no significant changes in synaptic RF gain $(101.9 \pm 13.8 \%$; $p=$ $0.4)$ (Table 1) or synaptic RF width (101.6 $\pm 15.7 \%$; $p=0.65)$ (Table 1) compared with those measured after adaptation to a gray screen.

\section{Intrinsic membrane properties may contribute to receptive field dynamics}

We have shown previously that the activation of a single cortical neuron with the intracellular injection of current can result in a hyperpolarization that can significantly reduce the visual response of a neuron to low-contrast drifting gratings (SanchezVives et al., 2000a). In addition, we have shown that this hyperpolarization may be mediated by the activation of intrinsic $\mathrm{K}^{+}$ conductances (Sanchez-Vives et al., 2000b). These results indicate that intrinsic membrane mechanisms can contribute to the phenomenon of contrast adaptation and its aftereffects. We have shown here that two aftereffects of contrast adaptation are significant reductions of both the size and gain of the discharge RF (Figs. 1-3, 8-10). We hypothesized that these aftereffects could also be mimicked by the activation of a single cortical neuron with intracellular current injection.

To test this hypothesis, we used a hybrid protocol that consisted of injecting sinusoidal $(2 \mathrm{~Hz})$ current in the recorded neuron for $10 \mathrm{~s}$ and of sufficient intensity to cause the cell to spike at $31.4 \pm 10.6 \mathrm{~Hz}$ (at the beginning of current injection), while its RF was continuously mapped with white and black bars. As observed previously (Sanchez-Vives et al., 2000a), intracellular injection of sinusoidal current was followed by a significant hyperpolarization of the membrane potential in 12 of 20 cells tested (Fig. $11 \mathrm{~B}$ ). The mean amplitude of this hyperpolarization was $-3.07 \pm 2.10 \mathrm{mV}$ (range, -6.48 to $-0.53 \mathrm{mV} ; n=12$ ). The time constant of recovery from this hyperpolarization was $6.9 \pm 3.6 \mathrm{~s}$ ( $n=5$ cells, with $r^{2}$ of exponential decay fit $>0.5$ ), a value very similar to the time constant of recovery after high-contrast adaptation (6.7 s; see above).

On the population level ( $n=10$ cells that satisfied our criteria of significance of visual response), the mean membrane potential 


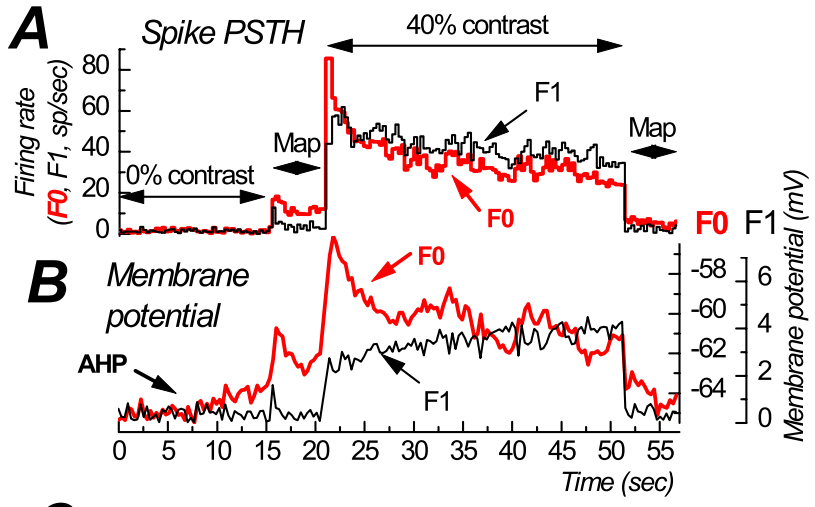

C Discharge receptive field, space-time plot
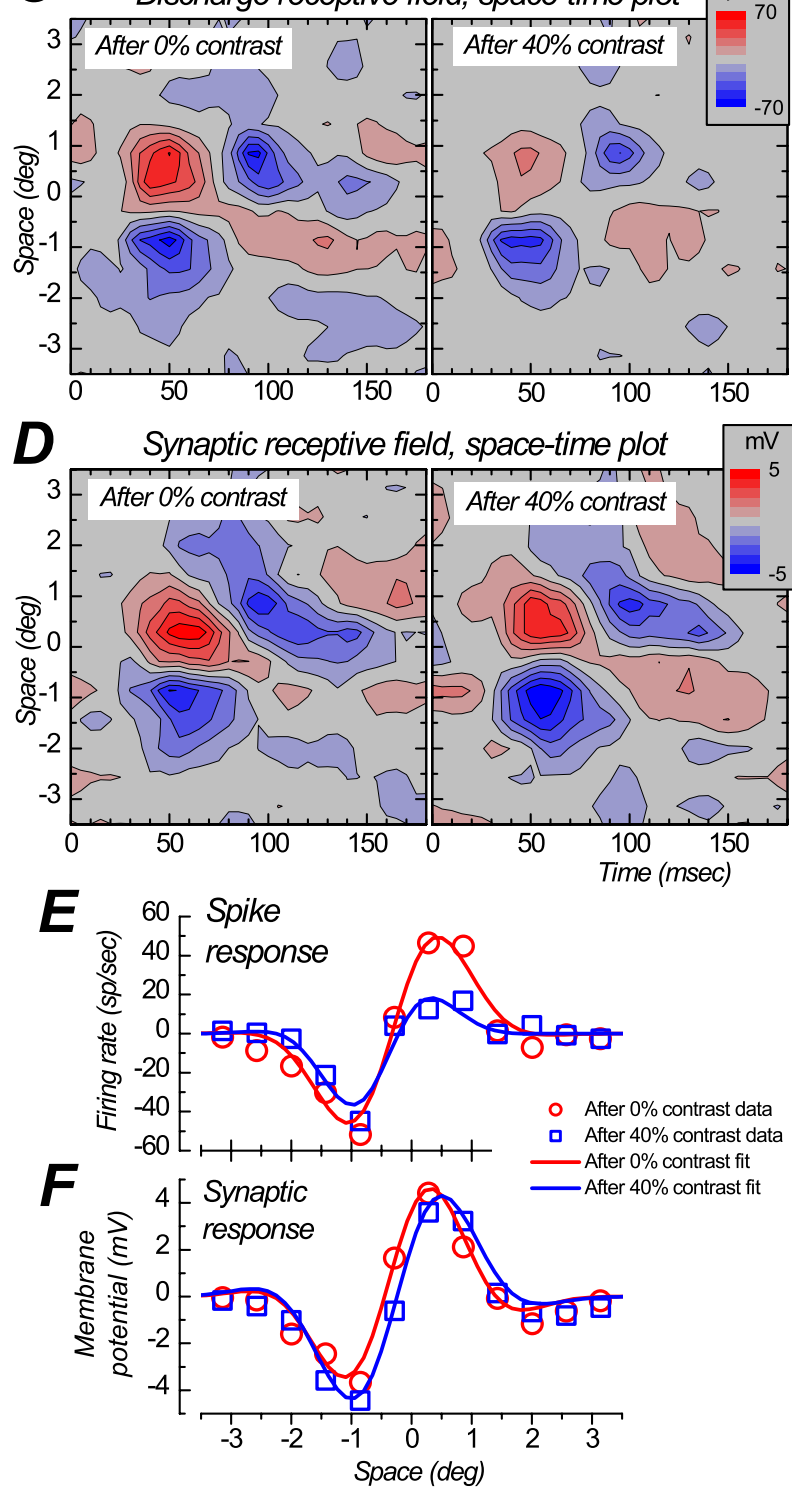

Figure 8. Aftereffects of high-contrast stimulation on the discharge and synaptic RFs in a simple cell. $A$, Spike response ( $F_{0}$ component, red; $F_{1}$ component, black) as a function of time shows that the neuron was strongly activated by the high-contrast stimulus. $B$, Membrane potential time series ( $F_{0}$ and $F_{1}$ components). Because stimuli sequences were given in a loop (17 repetitions), the $0 \%$ contrast period follows the map after the $40 \%$ contrast stimulation. The high-contrast stimulus induced a hyperpolarization of the membrane potential. This hyperpolarization ("AHP") slowly deactivated during the $0 \%$ contrast period, leading to the slow depolarization of the neuron during adaptation to the gray screen. C, Discharge RF maps obtained before (left) and after (right) high-contrast adaptation (periods labeled "Map" in A). Maps over the $5 \mathrm{~s}$ of mapping showed a difference ranging from -5.71 to $+1.43 \mathrm{mV}$ (average, $-1.55 \pm 1.85 ; p=0.02$ ) after the intracellular injection of current compared with that before current injection (Fig. 12 A; Table 1). This hyperpolarization was associated with a significant decrease in the discharge RF gain to $82.5 \pm$ $22.0 \%(p=0.03)$ (Fig. 12E; Table 1). At the single-cell level, this effect was significant in 4 of 10 cells (Table 1).

The hyperpolarization that followed the activation of the neuron with sinusoidal current injection was not associated with a significant decrease in discharge RF width $(93.8 \pm 13.9 \%$; $p=$ 0.14 ) (Fig. 12J; Table 1); only 2 of 10 cells showed a significant reduction. At the population level, synaptic RF gain and width were not modified in these protocols $(p=0.65$ and $p=0.88$, respectively) (Figs. $12 B, G$; Table 1 ).

Importantly, there is a significant correlation between the change in discharge RF gain and the amplitude of the hyperpolarization (Spearman's $\rho=0.94 ; p=0.005$ ) (Fig. 12C) such that cells that showed the largest hyperpolarization were those that showed the strongest reduction in firing rate. In addition, there was a significant correlation between membrane potential hyperpolarization and discharge RF width changes $(\rho=0.66 ; p=$ 0.047) (Fig. 12 H). This suggests that a long-lasting hyperpolarization generated through the intrinsic membrane properties of cortical neurons can contribute to the control of discharge RF width and gain.

\section{Discussion}

We demonstrated here that the gain, and to some degree the width, of cortical discharge RFs are strongly dependent on the adaptation state of the neuron. After adaptation to a highcontrast stimulus, there was a marked reduction in discharge RF gain and a lesser decrease in RF width. Conversely, deadaptation, as occurred during the subsequent presentation of a gray screen or artificial scotoma, showed the opposite effects: a large increase in discharge RF gain and a small increase in RF width. These changes were mediated in part by membrane potential changes that occurred with adaptation and de-adaptation. In a subpopulation of cells, these adaptation effects were also supported by changes in visually evoked synaptic potentials arriving in the recorded neurons. Finally, we found no significant differences between presentation of an artificial scotoma and a gray full screen, indicating that changes in gain and width are contingent on direct RF stimulation.

\section{Aftereffects of high-contrast stimulation on RF size and gain} Area 17 neurons receive synaptic inputs from a large region of visual space because of horizontal connections within area 17 (Gilbert and Wiesel, 1983; Martin and Whitteridge, 1984) and feedback connections from extrastriate visual areas (Salin et al., 1992; Angelucci et al., 2002). The amplitude of visually evoked synaptic activity peaks at the center and decreases toward the periphery of the RF (Grinvald et al., 1994; Das and Gilbert, 1995a; Toth et al., 1996; Bringuier et al., 1999). Thus, a change in the membrane potential of a cortical neuron should modify both the width and the gain of the discharge RF through an iceberg effect.

obtained after subtraction of dark bar response from bright bar response. D, Synaptic RF maps. The membrane potential during mapping was hyperpolarized by $1.8 \mathrm{mV}$ on average after highcontrast compared with after $0 \%$ contrast. $E$, Gabor fits through the spatial discharge RF map at the time of the peak response show strongly reduced gain (58\%) but no significant width reduction (87\%). F, Gabor fits through the peak synaptic response do not reveal a significant aftereffect of high-contrast adaptation on synaptic RF width (102\%) or gain (108\%). 


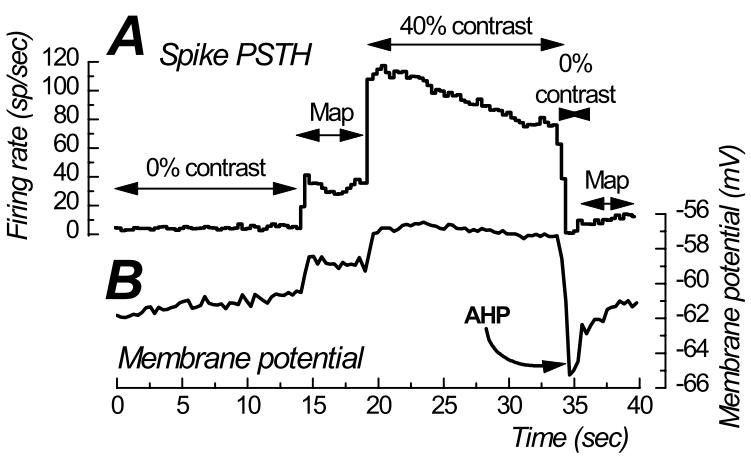

C Discharge receptive field, space-time plot

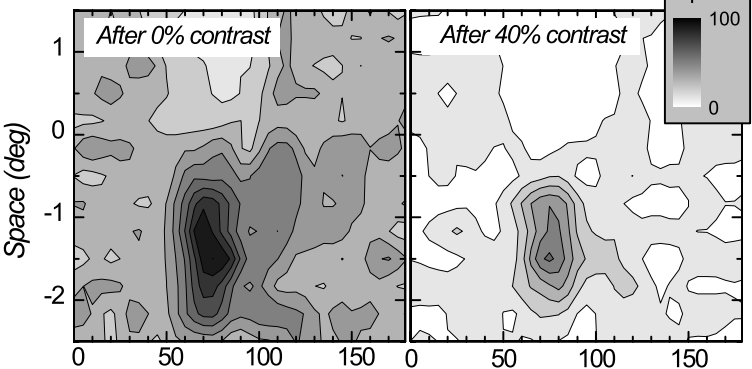

D
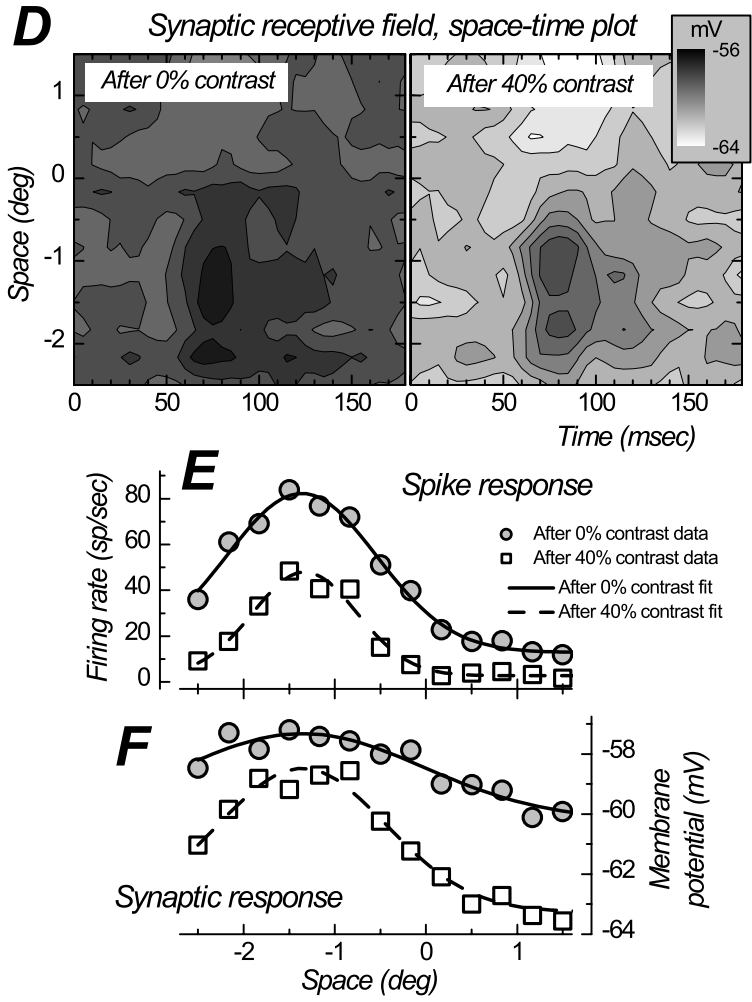

Figure 9. Aftereffects of high-contrast stimulation on the discharge and synaptic RFs in a complex cell. $A, B$, Spike response ( $F_{0}$ component) and membrane potential ( $F_{0}$ component) as a function of time. In this case, a $1 s$ duration gray screen stimulus was inserted between the end of the high-contrast and the beginning of the second RF mapping. This allowed for the full expression of the post-adaptation hyperpolarization ("AHP"). Because stimuli sequences were given in a loop (21 repetitions), the hyperpolarization was still visible and slowly lessened during the $0 \%$ contrast period, leading to the slow depolarization of the neuron during adaptation to the gray screen. C, D, Discharge and synaptic RF maps obtained before (left) and after (right) high-contrast adaptation. $x-t$ plots show dark bar response. The membrane potential during mapping was hyperpolarized by $2.9 \mathrm{mV}$ on average after high-contrast compared with after $0 \%$ contrast. $E$, Gaussian fits through the peak response of the discharge RF map show significant reduction in both gain (65\%) and width (69\%). F, Gaussian fits through the peak response of the synaptic RF map show significantly increased gain (167\%) and decreased width (69\%) after high contrast.
Effects of high contrast on discharge receptive field gain vs. membrane potential and synaptic receptive field gain

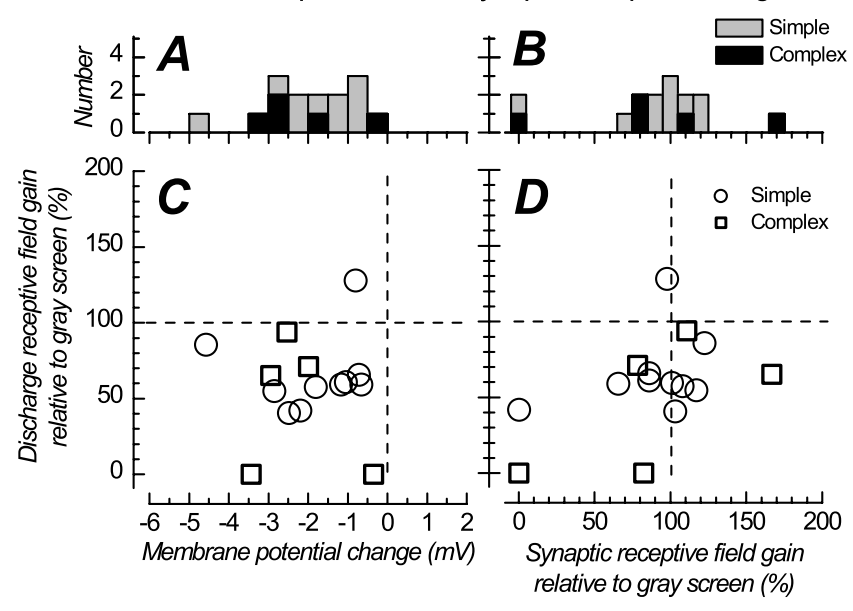

Effects of high contrast on discharge receptive field width vs. membrane potential and synaptic receptive field width
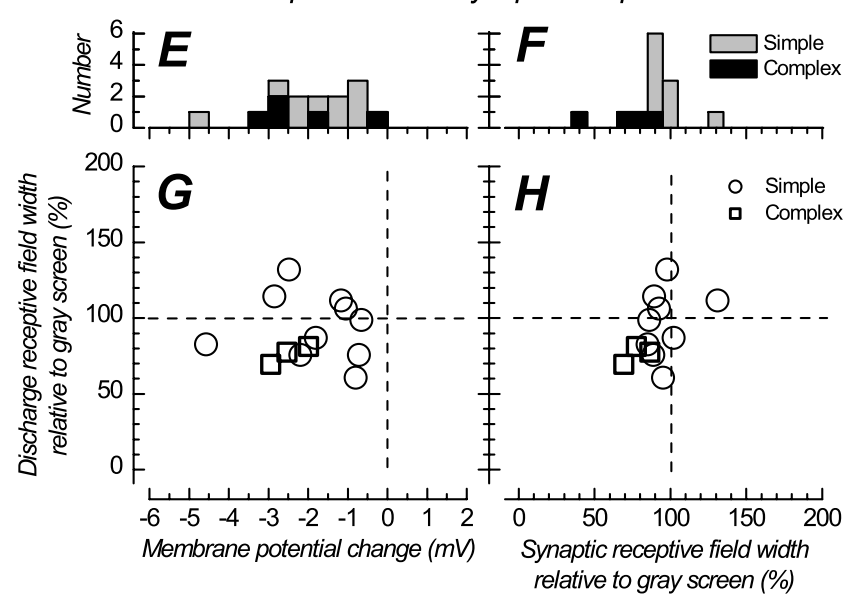

Figure 10. Impacts of membrane potential hyperpolarization and changes in synaptic RF on discharge RF gain and width after high-contrast adaptation. A, Histogram depicting the membrane potential hyperpolarization after high-contrast stimulation. The mean membrane potential during the mapping after high contrast has been subtracted from that during the mapping after $0 \%$ contrast stimulation. B, Histogram illustrating the gain of synaptic RF after high contrast, expressed as a percentage of the value obtained after $0 \%$ contrast stimulation. C, Relative gain of the discharge RF plotted against the change in membrane potential. D, Relative discharge RF gain plotted against relative synaptic RF gain. The simple cell that showed a synaptic RF gain down to 0 after high-contrast stimulation actually exhibited a significant discharge RF during this period. $E$, Membrane potential hyperpolarization after high-contrast stimulation (same as A).F, Relative synaptic RF width after high contrast. In contrast to synaptic RF gain, the synaptic RF width was significantly reduced after high contrast. G, Relative discharge RF width plotted against membrane potential change. $H$, Relative discharge RF width plotted against relative synaptic RF width. The decrease in synaptic RF width in simple cells was not systematically associated with a decrease in discharge RF width. To allow for a comparison with the aftereffects of sinusoidal current injection, the scales in $A-H$ are the same as in Figure 12.

In accordance with the iceberg effect, we found that hyperpolarization of single neurons by a few millivolts with the intracellular injection of current led to a significant reduction of discharge RF width and gain (Figs. 5-7). In contrast to the discharge $\mathrm{RF}$, the synaptic RF showed on average no significant change in width. However, when the cell was more hyperpolarized, the synaptic RF showed an increase in gain, which may be attributable to an enhanced driving force for excitatory synaptic potentials, combined with a decreased shunt of synaptic conductances when spike response was reduced. 

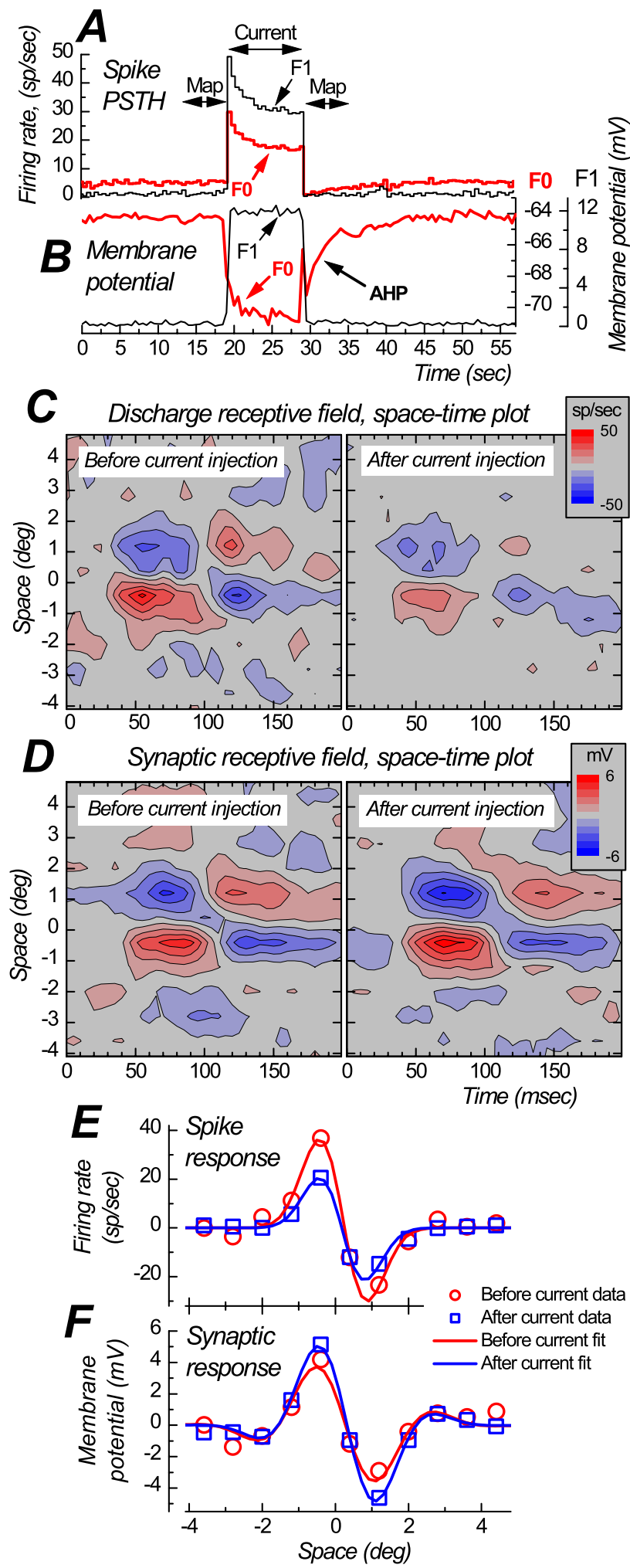

Figure 11. Aftereffects of action potential discharge induced by the intracellular injection of sinusoidal currents on discharge and synaptic RFs in a simple cell. The RF maps in ( and D were obtained immediately before (maps on the left) or after (maps on the right) the injection, for $10 \mathrm{~s}$, of a high-intensity (1.5 nA peak to peak) sinusoidal current in the recorded neuron $(A, B)$. To allow for averaging, the current injection has been repeated 28 times. A, PSTH. B, Membrane potential average. Both $F_{1}$ and $F_{0}$ components are shown. The current injection led to a hyperpolarization of the membrane potential ("AHP") that lasted for $\sim 15 \mathrm{~s}$ (time constant, $3.5 \mathrm{~s}$ ). The mapping stimuli were active at all times, but the maps shown in $($ and $D$ were calculated for $5 \mathrm{~s}$ before and $5 \mathrm{~s}$ after the current injection. $E$, RF profiles corresponding to the peak of the spike
The presentation of a high-contrast visual stimulus results in a 3-5 $\mathrm{mV}$ (on average) after hyperpolarization of the membrane potential (Carandini and Ferster, 1997; Sanchez-Vives et al., 2000a). Here we found that many cortical neurons exhibiting decreases in RF gain and width after high-contrast adaptation also exhibited a membrane potential hyperpolarization (Figs. $8-10)$. Neuronal firing induced with intracellular current injection also caused a subsequence hyperpolarization, presumably through the activation of $\mathrm{Na}^{+}$- and $\mathrm{Ca}^{2+}$-dependent $\mathrm{K}^{+}$currents (Schwindt et al., 1989; Sanchez-Vives et al., 2000b). Changes in the gain and width of the discharge RFs were correlated with this hyperpolarization (Fig. 12). This indicates that intrinsic membrane properties are potentially involved in the effects of visual adaptation on discharge RF properties.

In previous studies, it has been difficult to disentangle the contribution of intrinsic and synaptic mechanisms in contrast adaptation because of the continual nature of the visual stimuli (sine-wave gratings) (Ahmed et al., 1997; Carandini and Ferster, 1997; Sanchez-Vives et al., 2000a). In the present study, the use of localized flashed bars enabled us to separate intrinsic (membrane potential offset) and synaptic (synaptic RF gain and width) contribution to adaptation aftereffects in both simple and complex cells. Two observations indicate that synaptic mechanisms are involved in the aftereffects of high-contrast stimulation. First, in contrast to the nearly systematic increase obtained with DC injection, the synaptic RF gain showed a wider range of changes after high-contrast stimulation: from a complete suppression to a 1.7-fold increase (compare Fig. $10 \mathrm{D}$ with Fig. $7 \mathrm{C}$ ). Although increases or no changes may be consistent with an intrinsic mechanism, reduction and suppression of synaptic RF gain suggest that decreases in afferent activity also took place after highcontrast stimulation. Second, the synaptic RF width was also significantly reduced after high-contrast stimulation.

Contrast adaptation has both a nonspecific and a stimulusspecific component (Movshon and Lennie, 1979; Albrecht et al., 1984; Maddess et al., 1988; Hammond et al., 1989; Saul and Cynader, 1989; Carandini et al., 1997; Dragoi et al., 2000). Intrinsic mechanisms may underlie the nonspecific component of adaptation, whereas multiple synaptic mechanisms may be involved in the stimulus-specific component. Synaptic depression (Finlayson and Cynader, 1995; Varela et al., 1997; Chung et al., 2002) could be one of these, although synaptic depression might be weaker in vivo than in vitro (Kang et al., 1991; Sanchez-Vives et al., 1999; Boudreau and Ferster, 2003) (but see Chung et al., 2002). Changes in visually evoked synaptic responses may also be a consequence of neuronal interactions within the cortical network: the vast majority of excitatory synaptic contacts on all cortical neurons arises from other cortical cells (Binzegger et al., 2004); this organization may lead to the amplification of changes taking place at the single-cell level (Douglas and Martin, 1991). In this framework, membrane potential changes in populations of cells presynaptic to the recorded one would become manifest in modifications of its synaptic RF features.

response in the $x$ - $t$ plots in ( fitted with Gabor functions. No significant change in discharge RF width is evident $(96.5 \%)$, but gain reduction (66.5\%) during the afterhyperpolarization is clearly visible, indicating that activation of action potentials with current injection led to a decrease of response to the visual stimulation. $F$, The profiles and Gabor fits for the synaptic RFs indicate a significant gain increase (143\%) after sinusoidal current injection and no significant change in synaptic RF width (87\%). 
Effects and aftereffects of scotoma presentation

Presentation of artificial scotomas have been reported to cause a twofold to eightfold increase in RF area in cat V1 (Pettet and Gilbert, 1992; Das and Gilbert, 1995b), to increase response gain, without changing RF size, in many cat V1 cells (DeAngelis et al., 1995), or to have no effect on either RF size or gain in area V3 of the macaque monkey (De Weerd et al., 1995). Our results are similar to those of this last study: presenting an artificial scotoma did not have any additional effect on RF size and gain when compared with the effects of presenting a full gray screen.

Previous hypotheses to explain RF dynamics have proposed an adaptation of surround inhibition or a potentiation of horizontal connections by the prolonged scotoma presentation (Pettet and Gilbert, 1992; Xing and Gerstein, 1994; DeAngelis et al., 1995; Kalarickal and Marshall, 1999; Cavanaugh et al., 2002). Our results offer an alternative explanation, which is based on the adaptation state of the cells before RF measurements. Gilbert and colleagues found that, after expansion of the RF, direct visual stimulation of the neuron brought RF size back to the values measured before artificial scotoma presentation. The RFs at the beginning of the scotoma presentation were presumably in a contracted state because of previous visual stimulation of the neuron (Pettet and Gilbert, 1992). In addition, DeAngelis et al. (1995) showed that mapping RFs for an extended period might lead to a reduction of discharge receptive field gain. We have shown here that intense visual stimulation is followed by RF gain and width reduction. If the reference for size and gain were taken after intense visual stimulation, then the apparent changes brought on by long-lasting presentation of a scotoma would have the appearance of an increase in discharge RF gain and width. However, if the references for RF size and gain measurements are taken after a period of unpatterned stimulation, then scotoma presentation does not appear to further affect these RF properties. These observations suggest that it is merely the lack of direct stimulation of the cell during the scotoma presentation, and the progressive recovery from adaptation, that results in RF expansion.

Psychophysical studies (Kapadia et al., 1994; Tailby and Metha, 2004) showed that presentation of an artificial scotoma biases the perception of the position of target stimuli toward the scotoma center. This result have been interpreted as reflecting RF expansion for cells inside the scotoma. An alternative explanation, in closer accordance with our experimental data, is that the perceptual bias results from a decreased

\section{Effects of sinusoidal current injection on discharge receptive field gain vs. membrane potential and synaptic receptive field gain}
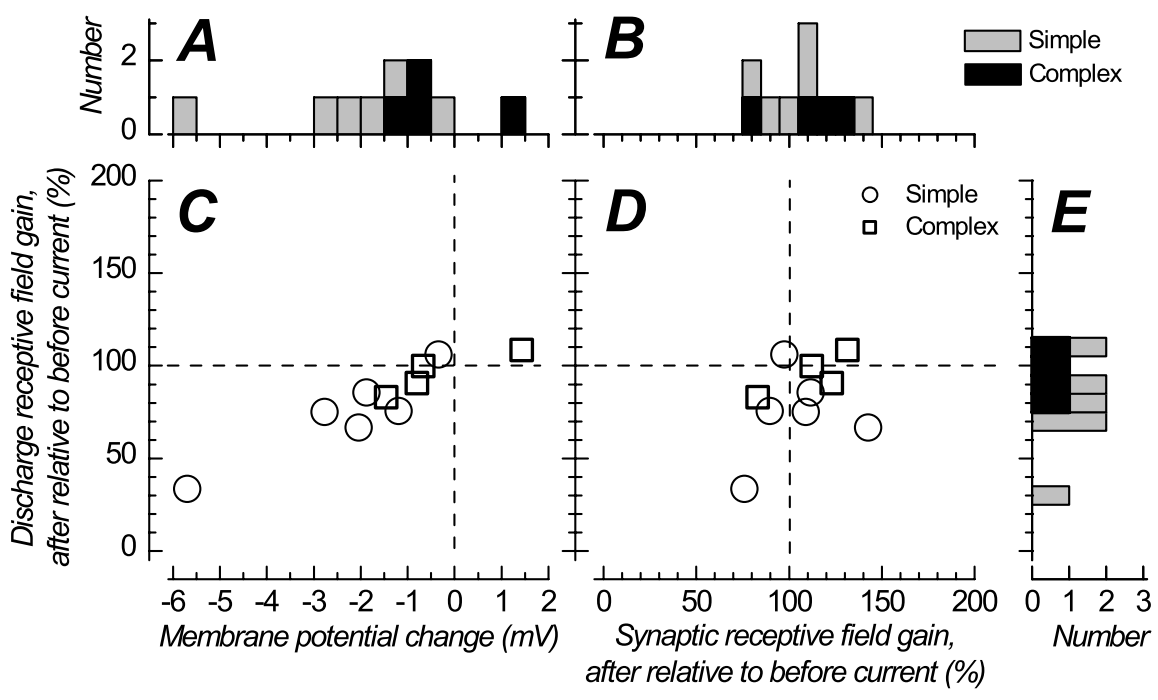

\section{Effects of sinusoidal current injection on discharge receptive field width vs. membrane potential and synaptic receptive field width}
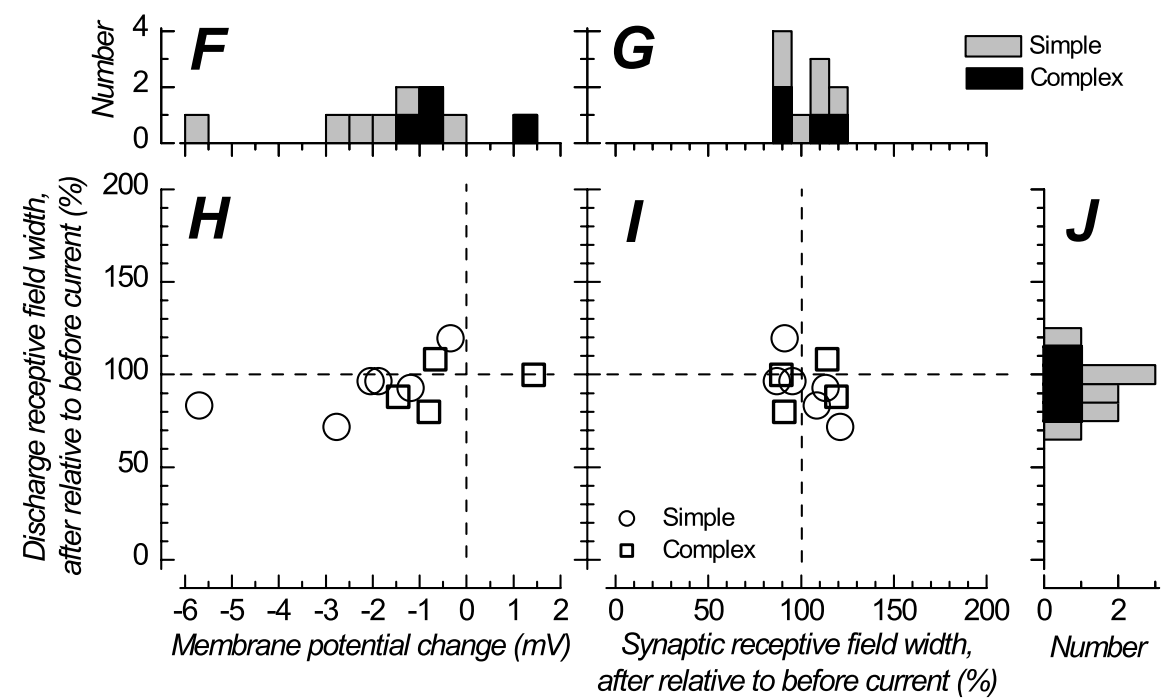

Figure 12. Consequences of high-intensity current injection on discharge and synaptic RF. $A$, Histogram showing that, at the population level, the membrane potential during the $5 \mathrm{~s}$ of mapping after intracellular sinusoidal current injection is more hyperpolarized than during the $5 \mathrm{~s}$ of mapping that precedes it. $B$, Synaptic RF gain after high-intensity current injection, expressed as a percentage of the value obtained before current injection. C, Relative discharge RF gain plotted against membrane potential change. There is a significant correlation between these two variables. $D$, Relative discharge RF gain plotted against percentage change in synaptic RF gain. $E$, Discharge RF gain, expressed as a percentage of the value obtained before current injection, is reduced, on average, after high-intensity current injection. $F$, Relative membrane potential after sinusoidal current injection replotted (same as $A$ ). G, Relative synaptic RF width after sinusoidal current injection, expressed as a percentage of the value obtained before current injection. $H$, Relative discharge $R F$ width plotted against membrane potential change. There is a significant correlation between these two variables. I, Relative discharge RF width plotted against relative synaptic RF width. J, Discharge RF width, expressed as a percentage of the value obtained before current injection, was not significantly reduced in these protocols. To allow for a comparison with the aftereffects of high-contrast stimulation, the scales are the same as in Figure 10. responsiveness as a result of adaptation for the cells near the scotoma border, whereas the non-adapted cells in the scotoma center would remain unaffected.

In humans, prolonged presentation of an artificial scotoma is accompanied by a progressive "filling-in" by the contents of the surrounding stimulus (Gerrits et al., 1966; Ramachandran and 
Gregory, 1991; De Weerd et al., 1998). In the monkey, neurons in areas V2 and V3 exhibit an increase in firing rate (so-called "climbing" activity) when their RF is covered by an artificial scotoma (De Weerd et al., 1995). Firing rates reach a plateau with a time course (a few seconds) that is similar to the time required for perceptual filling-in. The perceptual filling-in process has been hypothesized to result from adaptation to the border between the scotoma and the peripheral stimulus, followed by the propagation of the activity elicited by the peripheral stimulus toward the center of the scotoma (Gerrits and Vendrik, 1970; De Weerd et al., 1998). In our data, scotoma presentation did not result in increases in neuronal activity, consistent with a lack of climbing activity in V1. However, our demonstration of a prominent RF gain control by adaptation suggests that the first stage of filling-in may start taking place in V1 and may ultimately reside in the dynamic control of the membrane potential through intrinsic membrane properties.

\section{References}

Ahmed B, Allison JD, Douglas RJ, Martin KA (1997) An intracellular study of the contrast-dependence of neuronal activity in cat visual cortex. Cereb Cortex 7:559-570.

Albrecht DG, Farrar SB, Hamilton DB (1984) Spatial contrast adaptation characteristics of neurones recorded in cat's visual cortex. J Physiol (Lond) 347:713-739.

Anderson JS, Lampl I, Gillepsie DC, Ferster D (2001) Membrane potential and conductance changes underlying length tuning of cells in cat primary visual cortex. J Neurosci 21:2104-2112.

Angelucci A, Levitt JB, Walton EJ, Hupe JM, Bullier J, Lund JS (2002) Circuits for local and global signal integration in primary visual cortex. J Neurosci 22:8633-8646.

Binzegger T, Douglas RJ, Martin KA (2004) A quantitative map of the circuit of cat primary visual cortex. J Neurosci 24:8441-8453.

Boudreau CE, Ferster D (2003) Synaptic depression in thalamocortical synapses of the cat visual cortex. Soc Neurosci Abstr 29:484.11.

Bringuier V, Chavane F, Glaeser L, Fregnac Y (1999) Horizontal propagation of visual activity in the synaptic integration field of area 17 neurons. Science 283:695-699.

Carandini M, Ferster D (1997) A tonic hyperpolarization underlying contrast adaptation in cat visual cortex. Science 276:949-952.

Carandini M, Barlow HB, O’Keefe LP, Poirson AB, Movshon JA (1997) Adaptation to contingencies in macaque primary visual cortex. Philos Trans R Soc Lond B Biol Sci 352:1149-1154.

Cavanaugh JR, Bair W, Movshon JA (2002) Nature and interaction of signals from the receptive field center and surround in macaque $\mathrm{V} 1$ neurons. J Neurophysiol 88:2530-2546.

Chung S, Li X, Nelson SB (2002) Short-term depression at thalamocortical synapses contributes to rapid adaptation of cortical sensory responses in vivo. Neuron 34:437-446.

Das A, Gilbert CD (1995a) Long-range horizontal connections and their role in cortical reorganization revealed by optical recording of cat primary visual cortex. Nature 375:780-784

Das A, Gilbert CD (1995b) Receptive field expansion in adult visual cortex is linked to dynamic changes in strength of cortical connections. J Neurophysiol 74:779-792.

DeAngelis GC, Ohzawa I, Freeman RD (1993) Spatiotemporal organization of simple-cell receptive fields in the cat's striate cortex. I. General characteristics and postnatal development. J Neurophysiol 69:1091-1117.

DeAngelis GC, Freeman RD, Ohzawa I (1994) Length and width tuning of neurons in the cat's primary visual cortex. J Neurophysiol 71:347-374.

DeAngelis GC, Anzai A, Ohzawa I, Freeman RD (1995) Receptive field structure in the visual cortex: does selective stimulation induce plasticity? Proc Natl Acad Sci USA 92:9682-9686.

De Weerd P, Gattass R, Desimone R, Ungerleider LG (1995) Responses of cells in monkey visual cortex during perceptual filling-in of an artificial scotoma. Nature 377:731-734.

De Weerd P, Desimone R, Underleider LG (1998) Perceptual filling-in: a parametric study. Vision Res 38:2721-2734.

Douglas RJ, Martin KA (1991) A functional microcircuit for cat visual cortex. J Physiol (Lond) 440:735-769.
Dragoi V, Sharma J, Sur M (2000) Adaptation-induced plasticity of orientation tuning in adult visual cortex. Neuron 28:287-298.

Field DJ, Tolhurst DJ (1986) The structure and symmetry of simple-cell receptive field profiles in the cat's visual cortex. Proc R Soc Lond B Biol Sci 228:379-400.

Finlayson PG, Cynader MS (1995) Synaptic depression in visual cortex tissue slices: an in vitro model for cortical neuron adaptation. Exp Brain Res 106:145-155.

Gerrits HMJ, Vendrik AJH (1970) Simultaneous contrast, filling-in process and information processing in man's visual system. Exp Brain Res 11:411-430.

Gerrits HMJ, De Haan B, Vendrik AJH (1966) Experiments with retinal stabilized images. Relations between the observations and neural data. Vision Res 6:427-440.

Gilbert CD, Wiesel TN (1983) Clustered intrinsic connections in cat visual cortex. J Neurosci 3:1116-1133.

Grinvald A, Lieke EE, Frostig RD, Hildesheim R (1994) Cortical pointspread function and long-range lateral interactions revealed by real-time optical imaging of macaque monkey primary visual cortex. J Neurosci 14:2545-2568.

Hammond P, Pomfrett CJD, Ahmed B (1989) Neural motion after-effects in the cat's striate cortex: orientation selectivity. Vision Res 29:1671-1683.

Hartline HK (1938) The response of single optic nerve fibers of the vertebrate eye to illumination of the retina. Am J Physiol 121:400-415.

Heggelund P (1986) Quantitative study of the discharge fields of single cells in cat striate cortex. J Physiol (Lond) 373:277-292.

Hubel DH, Wiesel TN (1962) Receptive fields, binocular interaction and functional architecture in the cat's visual cortex. J Physiol (Lond) 160:106-154.

Jones JP, Palmer LA (1987a) The two-dimensional spatial structure of simple receptive fields in cat striate cortex. J Neurophysiol 58:1187-1211.

Jones JP, Palmer LA (1987b) An evaluation of the two-dimensional Gabor filter model of simple receptive fields in cat striate cortex. J Neurophysiol 58:1233-1258.

Kalarickal GJ, Marshall JA (1999) Models of receptive-field dynamics in visual cortex. Vis Neurosci 16:1055-1081.

Kang Y, Endo K, Araki T (1991) Differential connections by intracortical axon collaterals among pyramidal tract cells in the cat motor cortex. J Physiol (Lond) 435:243-256.

Kapadia MK, Gilbert CD, Westheimer G (1994) A quantitative measure for short-term cortical plasticity in human vision. J Neurosci 14:451-457.

Kapadia MK, Westheimer G, Gilbert CD (1999) Dynamics of spatial summation in primary visual cortex of alert monkeys. Proc Natl Acad Sci USA 96:12073-12078

Kitano M, Kasamatsu T, Norcia AM, Sutter EE (1995) Spatially distributed responses induced by contrast reversal in cat visual cortex. Exp Brain Res 104:297-309.

Kuffler SW (1953) Discharge patterns and functional organization of mammalian retina. J Neurophysiol 53:37-38.

Li C-Y, Li W (1994) Extensive integration field beyond the classical receptive field of cat's striate cortical neurons-classification and tuning properties. Vision Res 34:2337-2355.

Maddess T, McCourt ME, Blakeslee B, Cunningham RB (1988) Factors governing the adaptation of cells in area-17 of the cat visual cortex. Biol Cybern 59:229-236.

Maffei L, Fiorentini A, Bisti S (1973) Neural correlate of perceptual adaptation to gratings. Science 182:1036-1038.

Martin KAC, Whitteridge D (1984) Form, function and intracortical projections of spiny neurones in the striate visual cortex of the cat. J Physiol (Lond) 353:463-504.

McLean J, Palmer LA (1989) Contribution of linear spatiotemporal receptive field structure to velocity selectivity of simple cells in area 17 of cat. Vision Res 29:675-679.

Merrill EG, Ainsworth A (1972) Glass-coated platinum-plated tungsten microelectrodes. Med Biol Eng 10:662-672.

Monier C, Chavane F, Baudot P, Graham LJ, Fregnac Y (2003) Orientation and direction selectivity of synaptic inputs in visual cortical neurons: a diversity of combinations produces spike tuning. Neuron 37:663-680.

Movshon JA, Lennie P (1979) Pattern-selective adaptation in visual cortical neurones. Nature 278:850-852.

Movshon JA, Thompson ID, Tolhurst DJ (1978a) Spatial summation in the 
receptive fields of simple cells in the cat's striate cortex. J Physiol (Lond) 283:53-77.

Movshon JA, Thompson ID, Tolhurst DJ (1978b) Receptive field organization of complex cells in the cat's striate cortex. J Physiol (Lond) 283:79-99.

Nowak LG, Azouz R, Sanchez-Vives MV, Gray CM, McCormick DA (2003) Electrophysiological classes of cat primary visual cortical neurons in vivo as revealed by quantitative analysis. J Neurophysiol 89:1541-1566.

Palmer LA, Nafziger JS (2002) Effects of surround motion on receptive-field gain and structure in area 17 of the cat. Vis Neurosci 19:335-353.

Pettet MW, Gilbert CD (1992) Dynamic changes in receptive-field size in cat primary visual cortex. Proc Natl Acad Sci USA 89:8366-8370.

Ramachandran VS, Gregory RL (1991) Perceptual filling in of artificially induced scotomas in human vision. Nature 350:699-702.

Salin PA, Girard P, Kennedy H, Bullier J (1992) Visuotopic organization of corticocortical connections in the visual system of the cat. J Comp Neurol 320:415-434.

Sanchez-Vives MV, Nowak LG, McCormick DA (1999) Why might cortical synaptic depression be lesser in vivo than in vitro? Soc Neurosci Abstr 25:427.10.

Sanchez-Vives MV, Nowak LG, McCormick DA (2000a) Membrane mechanisms underlying contrast adaptation in cat area 17 in vivo. J Neurosci 20:4267-4285.

Sanchez-Vives MV, Nowak LG, McCormick DA (2000b) Cellular mechanisms of long-lasting adaptation in visual cortical neurons in vitro. J Neurosci 20:4286-4299.
Saul AB, Cynader MS (1989) Adaptation in single units in visual cortex: the tuning of aftereffects in the spatial domain. Vis Neurosci 2:593-607.

Sceniak MP, Ringach DL, Hawken MJ, Shapley R (1999) Contrast's effect on spatial summation by macaque V1 neurons. Nat Neurosci 2:733-739.

Schwindt PC, Spain WJ, Crill WE (1989) Long-lasting reduction of excitability by a sodium-dependent potassium current in cat neocortex neurons. J Neurophysiol 61:233-244.

Sengpiel F, Sen A, Blakemore C (1997) Characteristics of surround inhibition in cat area 17. Exp Brain Res 116:216-228.

Skottun B, DeValois RL, Grosof DH, Movshon JA, Albrecht DG, Bonds AB (1991) On classifying simple and complex cells according to response modulation. Vision Res 31:1079-1086.

Stevens JK, Gerstein GL (1976) Spatiotemporal organization of cat lateral geniculate receptive fields. J Neurophysiol 39:213-238.

Tailby C, Metha A (2004) Artificial scotoma-induced perceptual distortions are orientation dependent and short lived. Vis Neurosci 21:79-87.

Toth LJ, Rao SC, Kim D-S, Somers D, Sur M (1996) Subthreshold facilitation and suppression in primary visual cortex revealed by intrinsic signal imaging. Proc Natl Acad Sci USA 93:9869-9874.

Varela JA, Sen K, Gibson J, Fost J, Abbott LF, Nelson SB (1997) A quantitative description of short-term plasticity at excitatory synapses in layer $2 / 3$ of rat primary visual cortex. J Neurosci 17:7926-7940.

Xing J, Gerstein GL (1994) Simulation of dynamic receptive fields in primary visual cortex. Vision Res 14:1901-1911. 